\title{
Approximation of 3D Stokes flows in fractal domains
}

\author{
Massimo Cefalo, Simone Creo, Mirko Gallo
}

\begin{abstract}
We study a Stokes flow in a cylindrical-type fractal domain with homogeneous Dirichlet boundary conditions. We consider its numerical approximation by mixed methods: finite elements in space and finite differences in time. We introduce a suitably refined mesh à la Grisvard, which in turn will allow us to obtain an optimal a priori error estimate.
\end{abstract}

Keywords: Stokes equation, Fractals, Finite elements, Weighted Sobolev spaces.

2010 Mathematics Subject Classification: Primary: 35Q35, 65N12. Secondary: 35B65, 28A80.

\section{Introduction}

In this paper we study an incompressible Stokes equation, coupled with no-slip boundary conditions, in a three-dimensional cylindrical domain, which is the cartesian product between a two-dimensional pre-fractal domain of Koch type and the unit interval. This cylindrical set, which is polyhedral and non-convex, can model a rough microchannel.

${ }^{*}$ Dipartimento di Ingegneria Informatica, Automatica e Gestionale, Sapienza Università di Roma, Via Ariosto 25, 00185 Roma, Italy. E-mail: cefalo.m@gmail.com

${ }^{\dagger}$ Dipartimento di Scienze di Base e Applicate per l’Ingegneria, Sapienza Università di Roma, Via A. Scarpa 16, 00161 Roma, Italy. E-mail: simone.creo@sbai.uniroma1.it, mariarosaria.lancia@sbai.uniroma1.it, vernole@mat.uniroma1.it

${ }^{\ddagger}$ Dipartimento di Ingegneria Meccanica e Aerospaziale, Sapienza Università di Roma, Via Eudossiana 18, 00184 Roma, Italy. E-mail: mirko.gallo@uniroma1.it 
Nowadays, many papers deal with the study of viscous flows in rough micro-channels; this is due to the fast development of the so-called MEMS (which stands for Micro Electro-Mechanical Systems), e.g. micro-motors micro-turbines. Usually, Stokes equations model these phenomena.

The characteristics of the flow deeply affect the design and the process control of MEMS and heat transfer processes [31, 35, 37]. Fractal-type structures in the study of Stokes problems can be of great interest from the point of view of applications, firstly because they can model rough and irregular geometries, secondly because they could be important in the design of microfluidic devices. In fact, the mechanical stress exerted by the fluid onto solid boundaries is significant, when a large value of the velocity gradient towards the wall is achieved [33]. In this context, fractal-type geometries could mitigate these effects.

The study of Stokes problems in irregular domains is part of a long term project: the study of vector BVPs in fractal domains. This topic is rather recent and it is fast developing; for some literature on vector BVPs in irregular domains, we refer to $[1,20,21,27,8,9]$. As to the study of scalar BVPs in fractal-type domains, among the others we refer to $[26,25,11,13,10,12]$.

The main focus of this paper is on the numerical approximation of Stokes problems in fractal-type structures and on the proof of optimal a priori error estimates both in space and in time. We point out that the irregular nature of fractal-type boundaries does not allow us to use the standard techniques because the presence of irregular geometries deteriorates the regularity of the solution. For the numerical approximation of boundary value problems in fractal domains we refer to $[5,7,4]$.

More precisely, for every $n \in \mathbb{N}$, we consider parabolic incompressible Stokes problems $\left(\tilde{P}_{n}\right)$ in a cylindrical domain $Q_{n}$ with a Koch-type cross section (see Section 1), with homogeneous Dirichlet boundary conditions:

$$
\left(\tilde{P}_{n}\right) \begin{cases}\frac{\partial \mathbf{u}_{n}}{\partial t}(t, x)-\Delta \mathbf{u}_{n}(t, x)+\nabla p_{n}(t, x)=\mathbf{f}_{n}(t, x) & \text { in }[0, T] \times Q_{n}, \\ \operatorname{div} \mathbf{u}_{n}(t, x)=0 & \text { on }[0, T] \times Q_{n}, \\ \mathbf{u}_{n}(t, x)=0 & \text { on }[0, T] \times \partial Q_{n}, \\ \mathbf{u}_{n}(0, x)=\mathbf{u}_{n}^{0}(x) & \text { in } Q_{n} .\end{cases}
$$

Existence and uniqueness results for the weak solution $\mathbf{u}_{n}$ of $\left(\tilde{P}_{n}\right)$ and for its associated pressure $p_{n}$, for every $n \in \mathbb{N}$, are provided in [27], along with the corresponding results in the limit fractal domain $Q$ (obtained in the limit as $n \rightarrow+\infty$ ). Moreover, it is proved that the couple $(\mathbf{u}, p)$, given by the solution of the Stokes problem in the fractal domain and by its associated pressure, can be suitably approximated in terms of $\left(\mathbf{u}_{n}, p_{n}\right)$. The numerical approximation of problem $\left(\tilde{P}_{n}\right)$ is carried out by mixed methods, namely 
finite elements in space and finite differences in time. Since $Q_{n}$ is a non-convex domain, in order to prove an optimal a priori error estimate for the semi-discrete error, we construct a suitable mesh à la Grisvard [17], thus extending to the 3D case the results of [6]. We adapt to our case the regularity results in weighted Sobolev spaces for the couple $\left(\mathbf{u}_{n}, p_{n}\right)$ provided in [3], where a non-conforming method on pentahedral and tetrahedral meshes is obtained by using Crouziex-Raviart interpolation operators [14]. We then discretize the problem in time by an implicit method. We obtain optimal a priori error estimates by means of suitable discrete Sobolev-type norms (see $[19,18]$ ). We conclude by presenting some numerical simulations. Moreover, we prove that the average wall shear stress vanishes as $n \rightarrow+\infty$ for Hagen-Poiseuille-like flows.

The paper is organized as follows. In Section 1 we introduce the functional setting of this paper. In Section 2 we recall the existence and uniqueness results for the solution of problem $\left(P_{n}\right)$ and its associated pressure and we introduce two equivalent weak formulations, which allow us to give an "efficient" discrete version of the space of divergence-free functions. In Section 3 we introduce the weighted Sobolev spaces and we recall the regularity results for the couple $\left(\mathbf{u}_{n}, p_{n}\right)$. In Section 4 we prove that the average wall shear stress tends to zero as $n \rightarrow+\infty$. In Section 5 we approximate problem $\left(\tilde{P}_{n}\right)$ by FEM in space and FD in time and we prove optimal a priori error estimates. In Section 6 we present the numerical simulations of the problem at hand.

\section{Preliminaries}

We denote by $\left|P-P_{0}\right|$ the Euclidean distance between two points in $\mathbb{R}^{3}$. By the Koch snowflake $K$ we denote the union of three coplanar Koch curves $K_{i}$ (see [16]). We point out that $K$ has Hausdorff dimension $d_{f}=\frac{\ln 4}{\ln 3}$

We assume that the junction points $A_{1}, A_{3}$ and $A_{5}$ are the vertices of a regular triangle with unit side length, i.e. $\left|A_{1}-A_{3}\right|=\left|A_{1}-A_{5}\right|=\left|A_{3}-A_{5}\right|=1$. One can define, in a natural way, a finite Borel measure $\mu_{K}$ supported on $K$ by

$$
\mu_{K}:=\mu_{1}+\mu_{2}+\mu_{3}
$$

where $\mu_{i}$ denotes the normalized $d_{f}$-dimensional Hausdorff measure restricted to $K_{i}$, for $i=1,2,3$.

We denote by $K_{n}$ the pre-fractal closed polygonal curve approximating $K$ at the $n$-th step. We define $S_{n}=K_{n} \times I$, with $I=[0,1] ; S_{n}$ is a surface of polyhedral type. We give to a point $P \in S_{n}$ the Cartesian coordinates $P=\left(x, x_{3}\right)$, where $x=\left(x_{1}, x_{2}\right)$ are the coordinates of the orthogonal projection of $P$ on the plane containing $K_{n}$ and $x_{3}$ is the coordinate of the orthogonal projection of $P$ on the $x_{3}$-line containing the interval $I$. 
By $\Omega_{n} \subset \mathbb{R}^{2}$ we denote the open bounded two-dimensional domain with boundary $K_{n}$. By $Q_{n}=\Omega_{n} \times I$ we denote the cylindrical-type domain having $S_{n}$ as lateral surface and the sets $\Omega_{n} \times\{0\}$ and $\Omega_{n} \times\{1\}$ as bases.

The measure on $S_{n}$ is

$$
\mathrm{d} \sigma=\mathrm{d} \ell \times \mathrm{d} x_{3},
$$

where $\mathrm{d} \ell$ is the arc-length measure on $K_{n}$ (which can be naturally defined) and $\mathrm{d} x_{3}$ is the one-dimensional Lebesgue measure on $I$.

We introduce the fractal surface $S=K \times I$ given by the Cartesian product between $K$ and $I$. On $S$ we can define the finite Borel measure

$$
\mathrm{d} g=\mathrm{d} \mu_{K} \times \mathrm{d} x_{3} .
$$

We remark that $S$ has Hausdorff dimension $d_{f}+1$.

By $\Omega \subset \mathbb{R}^{2}$ we denote the two-dimensional fractal domain whose boundary is $K$. By $Q$ we denote the open cylindrical domain where $S=K \times I$ is the lateral surface and the sets $\Omega \times\{0\}$ and $\Omega \times\{1\}$ are the bases, see Figure 1 .

We point out that the sequence $\left\{Q_{n}\right\}$ is an increasing sequence invading $Q$, i.e.

$$
\mathcal{L}\left(Q \backslash Q_{n}\right) \underset{n \rightarrow+\infty}{\longrightarrow} 0
$$

where $\mathcal{L}$ is the Lesbegue measure in $\mathbb{R}^{3}$.

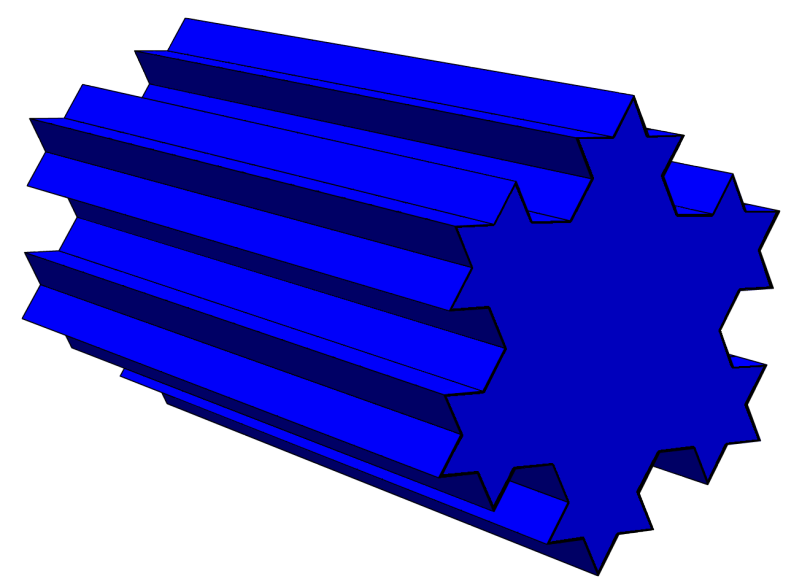

Figure 1: The fractal domain $Q$.

We now recall a trace theorem specialized to our case. For the proof we refer to Theorem 1 of Chapter VII in [23].

Theorem 1.1. Let $\Gamma$ denote $\Omega \times\{0\}$ and $\Omega \times\{1\}$. Then, for every $s>\frac{1}{2}, H^{s-\frac{1}{2}}(\Gamma)$ is the trace space of $H^{s}(Q)$, i.e.: 
1. there exists a linear and continuous operator $\gamma_{0}: H^{s}(Q) \rightarrow H^{s-\frac{1}{2}}(\Gamma)$;

2. there exists a linear and continuous operator Ext: $H^{s-\frac{1}{2}}(\Gamma) \rightarrow H^{s}(Q)$, such that $\gamma_{0} \circ$ Ext is the identity operator on $H^{s-\frac{1}{2}}(\Gamma)$.

Let $T$ be a compact set of $\mathbb{R}^{N}$. By $C(T)$ we denote the space of continuous functions on $T$ and by $C_{0}^{\infty}(T)$ the space of continuous infinitely differentiable functions with compact support in $T$.

Let $M$ be an open set of $\mathbb{R}^{3}$. By $L^{2}(M)$ we denote the Lebesgue space with respect to the Lebesgue measure $\mathcal{L}$. By $H^{1}(M)$ we denote the usual Sobolev space and by $\|\cdot\|_{H^{1}(M)}$ we denote its norm. We set $H_{0}^{1}(M)=\bar{C}_{0}^{\infty}(M) \|^{\| \cdot H_{H^{1}(M)}}$ and we denote by $H^{-1}(M)$ its dual.

In the following we define $H_{0}^{1}\left(Q_{n}\right)$ as the closure in $H^{1}(Q)$ of all functions $\phi \in C_{0}^{\infty}(Q)$ such that $\phi=0$ on $Q \backslash Q_{n}$.

We define $L^{2}(M)^{3}=\left\{\mathbf{u}=\left(u_{1}, u_{2}, u_{3}\right): u_{i} \in L^{2}(M)\right\}$ and $L_{\sigma}^{2}(M)={\overline{\left(C_{0, \sigma}^{\infty}(M)\right)}}^{L^{2}(M)^{3}}$, where $C_{0, \sigma}^{\infty}(M)=\left\{\mathbf{v} \in C_{0}^{\infty}(M)^{3}: \operatorname{div} \mathbf{v}=0\right\}$; we endow $L_{\sigma}^{2}(M)$ with the $L^{2}$-scalar product.

We introduce the space (see [32, page 25]):

$$
C_{0}^{\infty}\left([0, T) ; C_{0, \sigma}^{\infty}(M)\right):=\left\{\left.\mathbf{u}\right|_{[0, T) \times M}: \mathbf{u} \in C_{0, \sigma}^{\infty}((-1, T) \times M)^{3}, \operatorname{div} \mathbf{u}=0\right\}
$$

By $H_{0, \sigma}^{1}(M)={\overline{\left(C_{0, \sigma}^{\infty}(M)\right)}}^{H^{1}(M)}$ we denote the closed subspace of $H_{0}^{1}(M)^{3}$.

Moreover the following result holds (see Proposition 2.2. in [28]).

Proposition 1.2. The space $H_{0, \sigma}^{1}(M)$ is dense in $H_{0}^{1}(M)^{3}$.

We denote by $\mathcal{P}: L^{2}(M)^{3} \rightarrow L_{\sigma}^{2}(M)$ the Leray-Helmholtz projection and by $J$ the canonical injection $L_{\sigma}^{2}(M) \hookrightarrow L^{2}(M)^{3}$. We have that the adjoint of $J$ is $J^{\prime}=\mathcal{P}$ and that $\mathcal{P} \circ J$ is the identity on $L_{\sigma}^{2}(M)$. The canonical injection $\tilde{J}: H_{0, \sigma}^{1}(M) \hookrightarrow H_{0}^{1}(M)^{3}$ is the restriction of $J$ to $H_{0, \sigma}^{1}(M)$. We denote by $\tilde{\mathcal{P}}$ the adjoint of $\tilde{J}$; since $\tilde{J}$ is the restriction of $J$ to $H_{0, \sigma}^{1}(M), \tilde{\mathcal{P}}$ is an extension of $\mathcal{P}$ to $\left(H_{0, \sigma}^{1}(M)\right)^{\prime}$ (the dual of $H_{0, \sigma}^{1}(M)$ ). For more details on the Leray-Helmholtz projection operator see [32, Chapter II, Section 2.5].

\section{Existence and uniqueness results}

We now recall the main results about the Stokes problem in a pre-fractal domain, see [27]. 
The problem is formally stated as follows:

$$
\left(\tilde{P}_{n}\right) \begin{cases}\frac{\partial \mathbf{u}_{n}}{\partial t}(t, x)-\Delta \mathbf{u}_{n}(t, x)+\nabla p_{n}(t, x)=\mathbf{f}_{n}(t, x) & \text { in }[0, T] \times Q_{n}, \\ \operatorname{div} \mathbf{u}_{n}(t, x)=0 & \text { on }[0, T] \times Q_{n}, \\ \mathbf{u}_{n}(t, x)=0 & \text { on }[0, T] \times \partial Q_{n}, \\ \mathbf{u}_{n}(0, x)=\mathbf{u}_{n}^{0}(x) & \text { in } Q_{n} .\end{cases}
$$

We define for every $n \in \mathbb{N}$, the bilinear symmetric form $a_{n}(\mathbf{u}, \mathbf{v}): H_{0, \sigma}^{1}\left(Q_{n}\right) \times$ $H_{0, \sigma}^{1}\left(Q_{n}\right) \rightarrow \mathbb{R}:$

$$
a_{n}(\mathbf{u}, \mathbf{v})=\int_{Q_{n}} \nabla \mathbf{u} \cdot \nabla \mathbf{v} \mathrm{d} \mathcal{L} .
$$

We note that it is coercive in $H_{0, \sigma}^{1}\left(Q_{n}\right)$ thanks to Poincaré inequality and it is also closed in $L_{\sigma}^{2}\left(Q_{n}\right)$.

By Kato's theorem [24], there exists a unique non-positive self-adjoint operator $A_{0}^{n}: H_{0, \sigma}^{1}\left(Q_{n}\right) \rightarrow\left(H_{0, \sigma}^{1}\left(Q_{n}\right)\right)^{\prime}$ such that

$$
a_{n}(\mathbf{u}, \mathbf{v})=-\left\langle A_{0}^{n} \mathbf{u}, \mathbf{v}\right\rangle_{\left(H_{0, \sigma}^{1}\left(Q_{n}\right)\right)^{\prime}, H_{0, \sigma}^{1}\left(Q_{n}\right)} .
$$

Moreover, the following holds (see [28]).

Proposition 2.1. Let $\Delta: H_{0}^{1}\left(Q_{n}\right) \rightarrow H^{-1}\left(Q_{n}\right)$ denote the Dirichlet Laplacian on $Q_{n}$. There holds

$$
A_{0}^{n}=\tilde{\mathcal{P}} \circ(\Delta) \tilde{J}
$$

We call the Stokes operator $-A_{n}$ the part of $-A_{0}^{n}$ in $L_{\sigma}^{2}\left(Q_{n}\right)$, i.e. $D\left(A_{n}\right)=\{\mathbf{u} \in$ $\left.\left.H_{0, \sigma}^{1}\left(Q_{n}\right)\right): A_{0}^{n} \mathbf{u} \in L_{\sigma}^{2}\left(Q_{n}\right)\right\}$ and $A_{n} \mathbf{u}=A_{0}^{n} \mathbf{u}$.

Theorem 2.2. The operator $A_{n}$ is self-adjoint in $L_{\sigma}^{2}\left(Q_{n}\right)$ and generates an analytic contraction semigroup $T_{n}(t): L_{\sigma}^{2}\left(Q_{n}\right) \rightarrow D\left(A_{n}\right)$ with

$$
D\left(A_{n}\right)=\left\{\mathbf{u} \in H_{0, \sigma}^{1}\left(Q_{n}\right): \exists p \in\left(D\left(Q_{n}\right)\right)^{\prime} \text { s.t. } \nabla p \in H^{-1}\left(Q_{n}\right),-\Delta \mathbf{u}+\nabla p \in L_{\sigma}^{2}\left(Q_{n}\right)\right\}
$$

and

$$
-A_{n} \mathbf{u}=-\Delta \mathbf{u}+\nabla p \in L_{\sigma}^{2}\left(Q_{n}\right)
$$

Moreover, from [32, Chapter 3, Lemma 2.2.1], there exists a unique positive selfadjoint operator $\left(-A_{n}\right)^{\frac{1}{2}}: D\left(\left(-A_{n}\right)^{\frac{1}{2}}\right) \rightarrow L_{\sigma}^{2}\left(Q_{n}\right)$ with domain $D\left(\left(-A_{n}\right)^{\frac{1}{2}}\right)$ such that $D\left(-A_{n}\right) \subset D\left(\left(-A_{n}\right)^{\frac{1}{2}}\right) \subset L_{\sigma}^{2}\left(Q_{n}\right)$ and it enjoys the following properties:

$$
D\left(\left(-A_{n}\right)^{\frac{1}{2}}\right)=H_{0, \sigma}^{1}\left(Q_{n}\right) \quad \text { and } \quad\left\langle\left(-A_{n}\right)^{\frac{1}{2}} \mathbf{u},\left(-A_{n}\right)^{\frac{1}{2}} \mathbf{v}\right\rangle=\langle\nabla \mathbf{u}, \nabla \mathbf{v}\rangle .
$$


We now consider the following abstract Cauchy problem involving the operator $A_{n}$ :

$$
\left(\bar{P}_{n}\right)\left\{\begin{array}{l}
\frac{\partial \mathbf{u}_{n}}{\partial t}=A_{n} \mathbf{u}_{n}+\mathcal{P} \mathbf{f}_{n} \quad \text { in }(0, T) \\
\mathbf{u}_{n}(0)=\mathbf{u}_{n}^{0}
\end{array}\right.
$$

From Theorem 3.8 in [27], the following existence and uniqueness result for the solution of problem $\left(\bar{P}_{n}\right)$ holds.

Theorem 2.3. Let $\mathbf{f}_{n} \in L^{2}\left(0, T ; L^{2}\left(Q_{n}\right)^{3}\right)$ and $\mathbf{u}_{n}^{0} \in D\left(\left(-A_{n}\right)^{\frac{1}{2}}\right)$. For every $n \in \mathbb{N}$ we set

$$
\mathbf{u}_{n}(t)=T_{n}(t) \mathbf{u}_{n}^{0}+\int_{0}^{t} T_{n}(t-s) \mathcal{P} \mathbf{f}_{n}(s) \mathrm{d} s,
$$

where $T_{n}(t)$ is the analytic semigroup generated by $A_{n}$. Then $\mathbf{u}_{n}$ is the unique mild solution of $\left(\bar{P}_{n}\right)$, i.e.

$$
\begin{gathered}
\mathbf{u}_{n} \in H^{1}\left(0, T ; L_{\sigma}^{2}\left(Q_{n}\right)\right) \bigcap L^{2}\left(0, T ; D\left(A_{n}\right)\right), \\
\frac{\partial \mathbf{u}_{n}}{\partial t}(t)=A_{n} \mathbf{u}_{n}(t)+\mathcal{P} \mathbf{f}_{n}(t) \text { for almost every } t \in[0, T] \text { and } \mathbf{u}_{n}(0)=\mathbf{u}_{n}^{0}
\end{gathered}
$$

Moreover, there exists a constant $C$ independent from $n$ such that the following inequality holds:

$$
\left\|\mathbf{u}_{n}\right\|_{H^{1}\left(0, T ; L_{\sigma}^{2}\left(Q_{n}\right)\right)}+\left\|\mathbf{u}_{n}\right\|_{L^{2}\left(0, T ; D\left(A_{n}\right)\right)} \leq C\left\|\mathbf{f}_{n}\right\|_{L^{2}\left(0, T ; L^{2}\left(Q_{n}\right)^{3}\right)}+\left\|\mathbf{u}_{n}^{0}\right\|_{D\left(\left(-A_{n}\right)^{\frac{1}{2}}\right)^{.}}
$$

We now prove that the unique mild solution $\mathbf{u}_{n}$ of the abstract Cauchy problem $\left(\bar{P}_{n}\right)$ is also the unique weak solution of $\left(\tilde{P}_{n}\right)$. We recall the definition of weak solution and of associated pressure given in [32, Chapter IV, Definition 2.1.1].

Definition 2.4. Let $\mathbf{f}_{n} \in L^{2}\left(0, T ; L^{2}\left(Q_{n}\right)^{3}\right)$ and $\mathbf{u}_{n}^{0} \in L_{\sigma}^{2}\left(Q_{n}\right)$. A function $\mathbf{u}_{n} \in$ $L^{2}\left(0, T ; H_{0, \sigma}^{1}\left(Q_{n}\right)\right)$ is called a weak solution of the Stokes system $\left(\tilde{P}_{n}\right)$ if and only if

$$
-\int_{0}^{T} \int_{Q_{n}} \mathbf{u}_{n} \cdot \frac{\partial \mathbf{v}}{\partial t} \mathrm{~d} \mathcal{L} \mathrm{d} t+\int_{0}^{T} \int_{Q_{n}} \nabla \mathbf{u}_{n} \cdot \nabla \mathbf{v} \mathrm{d} \mathcal{L} \mathrm{d} t=\int_{Q_{n}} \mathbf{u}_{0} \cdot \mathbf{v}(0) \mathrm{d} \mathcal{L}+\int_{0}^{T} \int_{Q_{n}} \mathbf{f}_{n} \cdot \mathbf{v} \mathrm{d} \mathcal{L} \mathrm{d} t
$$

for every $\mathbf{v} \in C_{0}^{\infty}\left([0, T) ; C_{0, \sigma}^{\infty}\left(Q_{n}\right)\right)$. A distribution $p_{n}$ in $[0, T) \times Q_{n}$ is called an associated pressure of a weak solution $\mathbf{u}_{n}$ if and only if

$$
\frac{\partial \mathbf{u}_{n}}{\partial t}(t, x)-\Delta \mathbf{u}_{n}(t, x)+\nabla p_{n}(t, x)=\mathbf{f}_{n}(t, x)
$$

is satisfied in the sense of distributions. 
Theorem 2.5. For each $n \in \mathbb{N}$, if $\mathbf{f}_{n} \in L^{2}\left(0, T ; L^{2}\left(Q_{n}\right)^{3}\right)$ and $\mathbf{u}_{n}^{0} \in L_{\sigma}^{2}\left(Q_{n}\right)$, then the unique mild solution $\mathbf{u}_{n}$ of problem $\left(\bar{P}_{n}\right)$ given by (2.3) is the unique weak solution of the Stokes system $\left(\tilde{P}_{n}\right)$ in the sense of Definition 2.4. Moreover, there exists a unique pressure $p_{n} \in L^{2}\left(0, T ; L^{2}\left(Q_{n}\right)\right)$ associated to the solution $\mathbf{u}_{n}$ such that, for a.e. $t \in[0, T]$

$$
\int_{Q_{n}} p_{n}(t, x) \mathrm{d} \mathcal{L}=0
$$

For the proof, see Theorem 3.11 and Section 3.3 in [27].

We now introduce an equivalent weak formulation for $\left(\tilde{P}_{n}\right)$ in view of the numerical approximation of the pre-fractal problems by mixed methods, i.e. FEM in space and finite differences in time. We refer to [30] and [36] for details.

We point out that the space $H_{0, \sigma}^{1}\left(Q_{n}\right)$ coincides with the space

$$
V:=\left\{\mathbf{u} \in H_{0}^{1}\left(Q_{n}\right)^{3}: \operatorname{div} \mathbf{u}=0\right\}
$$

for bounded Lipschitz domains (see Lemma 1.2.1, page 111 in [32]). Moreover, from Lemma 2.5.3, page 82 in [32] we have that $L_{\sigma}^{2}\left(Q_{n}\right)=\left\{\mathbf{u} \in L^{2}\left(Q_{n}\right)^{3}: \operatorname{div} \mathbf{u}=0, \nu\right.$. $\left.\left.\mathbf{u}\right|_{\partial Q_{n}}=0\right\}$.

Let $\mathbf{u}_{n} \in V$ be the weak solution of $\left(\bar{P}_{n}\right)$. We multiply the first equation in $\left(\bar{P}_{n}\right)$ by a function $\mathbf{v} \in V$ not depending on time and we integrate on $Q_{n}$. By using the following property of the Leray-Helmholtz projection (see Lemma 2.5.2, Chapter II in [32])

$$
(\mathrm{Id}-\mathcal{P}) \mathbf{f}=\nabla p
$$

and integrating by parts, we obtain for a.e. $t \in[0, T)$

$$
\int_{Q_{n}} \frac{\partial \mathbf{u}_{n}}{\partial t} \cdot \mathbf{v} \mathrm{d} \mathcal{L}+\int_{Q_{n}} \nabla \mathbf{u}_{n} \cdot \nabla \mathbf{v} \mathrm{d} \mathcal{L}=\int_{Q_{n}} \mathbf{f}_{n} \cdot \mathbf{v} \mathrm{d} \mathcal{L} .
$$

The existence and uniqueness of the weak solution in $V$ of this problem (which we still denote by $\mathbf{u}_{n}$ ) follows from [15, Chapter XIX, Section 2].

This functional setting is not convenient for the numerical approximation of $\left(\tilde{P}_{n}\right)$. Indeed, it is difficult to find a finite dimensional space of divergence-free vector valued functions which allows us to find a good convergence behavior of the approximation error. Moreover, it can be very complicated to construct a basis of this finite dimensional space of divergence-free functions.

We then consider an alternative weak formulation of the Stokes system $\left(\tilde{P}_{n}\right)$. We 
introduce the space

$$
\mathcal{Q}=\left\{v \in L^{2}\left(Q_{n}\right): \int_{Q_{n}} v \mathrm{~d} \mathcal{L}=0\right\} .
$$

By multiplying the first and the second equations of $\left(\tilde{P}_{n}\right)$ by a function $\mathbf{v} \in H_{0}^{1}\left(Q_{n}\right)^{3}$ and integrating on $Q_{n}$, we get the following weak formulation of $\left(\tilde{P}_{n}\right)$ : for a.e. $t \in[0, T)$, given $\mathbf{f}_{n} \in L^{2}\left(Q_{n}\right)^{3}$ and $\mathbf{u}_{n}^{0} \in V$, find $\mathbf{u}_{n} \in H_{0}^{1}\left(Q_{n}\right)^{3}$ and $p_{n} \in \mathcal{Q}$ such that

$\left(P_{n}\right) \begin{cases}\frac{\partial}{\partial t} \int_{Q_{n}} \mathbf{u}_{n} \cdot \mathbf{v} \mathrm{d} \mathcal{L}+\int_{Q_{n}} \nabla \mathbf{u}_{n} \cdot \nabla \mathbf{v} \mathrm{d} \mathcal{L}-\int_{Q_{n}} p_{n} \operatorname{div} \mathbf{v} \mathrm{d} \mathcal{L}=\int_{Q_{n}} \mathbf{f}_{n} \cdot \mathbf{v} \mathrm{d} \mathcal{L} & \forall \mathbf{v} \in H_{0}^{1}\left(Q_{n}\right)^{3}, \\ \int_{Q_{n}} q \operatorname{div} \mathbf{u}_{n} \mathrm{~d} \mathcal{L}=0 & \forall q \in \mathcal{Q}, \\ \mathbf{u}_{n}(0, x)=\mathbf{u}_{n}^{0}(x) & \text { on } Q_{n} .\end{cases}$

We point out that the second equation in $\left(P_{n}\right)$ implies that $\operatorname{div} \mathbf{u}_{n}=0$ almost everywhere. Problem $\left(P_{n}\right)$ admits a unique weak solution $\mathbf{u}_{n} \in H_{0}^{1}\left(Q_{n}\right)^{3}$ and an associate pressure $p_{n} \in \mathcal{Q}$ (see [15, Chapter XIX]). The solution $\left(\mathbf{u}_{n}, p_{n}\right)$ of (2.6) is also a solution of (2.5) provided that the conditions of [27, Section 3.3] hold. The converse is also true (see [30, Chapters 10 and 13]).

\section{$3 \quad$ Regularity in weighted Sobolev spaces}

We now focus on proving regularity results in weighted Sobolev spaces for the couple $\left(\mathbf{u}_{n}, p_{n}\right)$, where $\mathbf{u}_{n}$ is the weak solution of problem $\left(P_{n}\right)$ and $p_{n}$ is its associated pressure. Such regularity results are needed for the numerical approximation of $\left(P_{n}\right)$; indeed, since the domain $Q_{n}$ is not convex, we have to construct a suitable triangulation of our domain for obtaining an optimal rate of convergence of the approximation error. The construction of the mesh will rely on this weighted Sobolev regularity. We recall that the interior angles in $Q_{n}$ have opening equal to $\frac{\pi}{3}$ or $\frac{4}{3} \pi$.

Following the notations of [3], for $\beta \in \mathbb{R}$ and $l \in \mathbb{N}$, we introduce the weighted Sobolev space $V_{\beta}^{l}\left(Q_{n}\right)$ of functions for which the following norm is finite:

$$
\|u\|_{V_{\beta}^{l}\left(Q_{n}\right)}^{2}:=\sum_{|k| \leq l} \int_{Q_{n}} r^{2(\beta-l+|k|)}\left|D^{k} u\right|^{2} \mathrm{~d} \mathcal{L},
$$

where $r=r(x)$ is the distance of $x \in Q_{n}$ from the edges.

It is known in literature (see e.g. [29]) that the regularity of the solution of Stokes problems in polyhedral domains is related to the smallest positive solution $\lambda$ of the 
following equation:

$$
\sin (\lambda \omega)=-\lambda \sin \omega
$$

where $\omega$ is the opening of the "worst" interior angle at the edge.

We rephrase equation (3.1) in our setting, by recalling that for $Q_{n} \omega=\frac{4}{3} \pi$. Let $\lambda>0$ be the (unique) positive solution of the equation

$$
\sin \left(\lambda \frac{4 \pi}{3}\right)=\lambda \frac{\sqrt{3}}{2}
$$

numerically, $\lambda \sim 0.61572$. From Theorem 2.1 in [3] the following result holds.

Theorem 3.1. Let $\left(\mathbf{u}_{n}, p_{n}\right)$ be the solution of $\left(P_{n}\right)$ and its associated pressure. Then for a.e. $t \in[0, T) \mathbf{u}_{n} \in V_{\beta}^{2}\left(Q_{n}\right)^{3}, p_{n} \in V_{\beta}^{1}\left(Q_{n}\right)$ for every $\beta \in(1-\lambda, 1), \partial_{x_{3}} \mathbf{u}_{n} \in V_{0}^{1}\left(Q_{n}\right)^{3}$ and $\partial_{x_{3}} p_{n} \in L^{2}\left(Q_{n}\right)$. Moreover, the following a priori estimate holds:

$$
\left\|\mathbf{u}_{n}\right\|_{V_{\beta}^{2}\left(Q_{n}\right)^{3}}+\left\|\partial_{x_{3}} \mathbf{u}_{n}\right\|_{V_{0}^{1}\left(Q_{n}\right)^{3}}+\left\|p_{n}\right\|_{V_{\beta}^{1}\left(Q_{n}\right)}+\left\|\partial_{x_{3}} p_{n}\right\|_{L^{2}\left(Q_{n}\right)} \leq C\left\|\mathbf{f}_{n}\right\|_{L^{2}\left(Q_{n}\right)},
$$

where $C$ depends on $Q_{n}$ and $\beta$.

Moreover, the following regularity result in fractional Sobolev spaces holds. For the proof, we refer to Proposition 4.15 in [22].

Theorem 3.2. Let $\left(\mathbf{u}_{n}, p_{n}\right)$ be as in Theorem 3.1. Then $\mathbf{u}_{n} \in H^{2-\beta}\left(Q_{n}\right)^{3}$ and $p_{n} \in$ $H^{1-\beta}\left(Q_{n}\right)$ for every $\beta \in(1-\lambda, 1)$.

\section{Mean shear stress}

Aim of this section is to prove that the average wall shear stress vanishes along the fractal boundary. For the sake of simplicity, we will confine ourselves to the stationary case and we will focus on an isolated system, where the external force contributions vanish. Under the latter assumption the balance force reads

$$
\int_{\partial Q_{n}} \mathbf{t}(\hat{\mathbf{n}}) \mathrm{d} \mathcal{S}=0,
$$

where $\mathbf{t}$ is the stress vector depending on the unit-length direction vector $\hat{\mathbf{n}}$. Due to the Cauchy's stress theorem, the vector $\mathbf{t}$ can be written in terms of a symmetric second-order tensor field $\mathbf{T}$ as $\mathbf{t}(\hat{\mathbf{n}})=\mathbf{T} \cdot \hat{\mathbf{n}}$, leading to

$$
\int_{\partial Q_{n}} \mathbf{T} \cdot \hat{\mathbf{n}} \mathrm{d} \mathcal{S}=0 .
$$


Since $\partial Q_{n}=S_{n} \cup\left(\Omega_{n} \times\{0\}\right) \cup\left(\Omega_{n} \times\{1\}\right)$ the above integral factorizes as

$$
\int_{S_{n}} \mathbf{T} \cdot \hat{\mathbf{n}} \mathrm{d} \sigma+\int_{\Omega_{n} \times\{0\}} \mathbf{T} \cdot \hat{\mathbf{n}} \mathrm{d} x_{1} \mathrm{~d} x_{2}+\int_{\Omega_{n} \times\{1\}} \mathbf{T} \cdot \hat{\mathbf{n}} \mathrm{d} x_{1} \mathrm{~d} x_{2}=0 .
$$

For Newtonian incompressible fluids, the stress tensor can be written as $\mathbf{T}=-p \mathbf{1}+$ $\mu\left(\nabla \mathbf{u}+\nabla \mathbf{u}^{T}\right)$, where $\mathbf{1}$ is the identity tensor, $p$ is the hydrodynamic pressure and $\mu$ is the viscosity coefficient. Clearly, in the latter case, the force balance is established between the viscous forces and the pressure field.

A particularly impressive form of the above balance is obtained when considering a Hagen-Poiseuille-like flow, where the cylindrical surface $Q_{n}$ has as predominant dimension the one represented by its axis. Hence, by denoting as $u_{\perp, n}$ the velocity component aligned with the cylindric axis, and with $\left(x_{1}, x_{2}\right)$ the coordinates in a generic section perpendicular to the axis, the solution $\mathbf{u}_{n}$ of the Stokes problem and its associated pressure $p_{n}$ take the simplified form (see e.g. [34])

$$
\mathbf{u}_{n}=\left(0,0, u_{\perp, n}\left(x_{1}, x_{2}\right)\right), \quad p_{\perp, n}\left(x_{3}\right)=p_{n}\left(0,0, x_{3}\right)
$$

We formally define by

$$
\left\langle\tau_{w, n}\right\rangle_{K_{n}}:=\frac{1}{\left|K_{n}\right|} \oint_{K_{n}} \mu \nabla u_{\perp, n} \cdot \hat{\mathbf{n}} \mathrm{d} \ell
$$

the "average wall shear stress". The following result holds.

Theorem 4.1. Let $\tilde{p}_{n}$ denote the trivial extension of $p_{n}$ to $Q$ and $\beta \in\left(1-\lambda, \frac{1}{2}\right)$. Let us suppose that there exists a constant $M>0$ independent from $n$ such that

$$
\left\|\tilde{p}_{n}\left(0,0, x_{3}\right)\right\|_{H^{1-\beta}(Q)} \leq M
$$

Then there exists a positive constant $C$ independent from $n$ such that

$$
\left|\left\langle\tau_{w, n}\right\rangle_{K_{n}}\right| \leq C \frac{4}{\sqrt[4]{3}}\left(\frac{3}{4}\right)^{n}|\Omega|
$$

hence the average wall shear stress $\left\langle\tau_{w, n}\right\rangle_{K_{n}}$ vanishes as $n \rightarrow+\infty$.

Proof. Under the above assumptions, the balance in (4.3) takes the form

$$
\int_{I} \mathrm{~d} x_{3} \oint_{K_{n}} \mu \nabla u_{\perp, n} \cdot \hat{\mathbf{n}} \mathrm{d} \ell+\int_{\Omega_{n} \times\{0\}} p_{\perp, n}\left(x_{3}\right) \mathrm{d} x_{1} \mathrm{~d} x_{2}-\int_{\Omega_{n} \times\{1\}} p_{\perp, n}\left(x_{3}\right) \mathrm{d} x_{1} \mathrm{~d} x_{2}=0 .
$$


From Theorem 3.1 all the above integrals are well defined for the solution $\mathbf{u}_{n}$ and its associated pressure $p_{n}$. By denoting with $\tau_{w, n}=\mu \nabla u_{\perp, n} \cdot \hat{\mathbf{n}}$ the wall shear stress, since $I=[0,1]$, one has

$$
\oint_{K_{n}} \tau_{w, n} \mathrm{~d} \ell+\left|\Omega_{n}\right|\left(\left.p_{\perp, n}\right|_{\left\{x_{3}=0\right\}}-\left.p_{\perp, n}\right|_{\left\{x_{3}=1\right\}}\right)=0,
$$

where $|\mathcal{A}|$ denotes the Lebesgue measure of a subset $\mathcal{A} \subset \mathbb{R}^{N}$.

By dividing both members of the above equation by $\left|K_{n}\right|$, we obtain

$$
\left\langle\tau_{w, n}\right\rangle_{K_{n}}=\frac{1}{\left|K_{n}\right|} \oint_{K_{n}} \tau_{w, n} \mathrm{~d} \ell=-\frac{\left|\Omega_{n}\right|}{\left|K_{n}\right|}\left(\left.p_{\perp, n}\right|_{\left\{x_{3}=0\right\}}-\left.p_{\perp, n}\right|_{\left\{x_{3}=1\right\}}\right)=\left(\frac{3}{4}\right)^{n}\left|\Omega_{n}\right|\left(\left.p_{\perp, n}\right|_{\left\{x_{3}=1\right\}}-\left.p_{\perp, n}\right|_{\left\{x_{3}=0\right\}}\right) \text {. }
$$

Since $\left|\Omega_{n}\right| \leq|\Omega|$, from (4.6) we obtain

$$
\left|\left\langle\tau_{w, n}\right\rangle_{K_{n}}\right| \leq\left(\frac{3}{4}\right)^{n}|\Omega|\left(\left.\left|p_{\perp, n}\right|_{\left\{x_{3}=1\right\}}|+| p_{\perp, n}\right|_{\left\{x_{3}=0\right\}} \mid\right) .
$$

We recall that from Theorem $3.2 p_{n} \in H^{1-\beta}\left(Q_{n}\right)$ for every $\beta \in(1-\lambda, 1)$; hence $\tilde{p}_{n} \in H^{1-\beta}(Q)$ for every $\beta \in(1-\lambda, 1)$.

From Sobolev embeddings and the trace Theorem 1.1, we obtain for every $\beta \in\left(1-\lambda, \frac{1}{2}\right)$ that

$$
\begin{aligned}
& \left.p_{\perp, n}\right|_{\left\{x_{3}=0\right\}}=\frac{1}{\left|\Omega_{n}\right|} \int_{\Omega_{n} \times\{0\}} p_{\perp, n}\left(x_{3}\right) \mathrm{d} x_{1} \mathrm{~d} x_{2} \leq \frac{1}{\left|\Omega_{n}\right|^{\frac{1}{2}}}\left\|p_{\perp, n}\left(x_{3}\right)\right\|_{L^{2}\left(\Omega_{n} \times\{0\}\right)}=\frac{1}{\left|\Omega_{n}\right|^{\frac{1}{2}}}\left\|p_{n}\left(0,0, x_{3}\right)\right\|_{L^{2}\left(\Omega_{n} \times\{0\}\right)} \\
& =\frac{1}{\left|\Omega_{n}\right|^{\frac{1}{2}}}\left\|\tilde{p}_{n}\left(0,0, x_{3}\right)\right\|_{L^{2}(\Omega \times\{0\})} \leq \frac{C_{\mathrm{Sob}}}{\left|\Omega_{n}\right|^{\frac{1}{2}}}\left\|\tilde{p}_{n}\left(0,0, x_{3}\right)\right\|_{H^{\frac{1}{2}-\beta}(\Omega \times\{0\})} \leq \frac{C_{\mathrm{Sob}} C_{\mathrm{Tr}}}{\left|\Omega_{n}\right|^{\frac{1}{2}}}\left\|\tilde{p}_{n}\left(0,0, x_{3}\right)\right\|_{H^{1-\beta}(Q)} .
\end{aligned}
$$

Since the area of $\Omega_{n}$ is bigger than the area of the equilateral triangle of vertices $A_{1}$, $A_{3}$ and $A_{5}$ (see Section 1), which in particular is $\frac{\sqrt{3}}{4}$, from (4.4), we have that

$$
\left.p_{\perp, n}\right|_{\left\{x_{3}=0\right\}} \leq C \frac{2}{\sqrt[4]{3}}
$$

where the constant $C$ depends on $Q$ and $M$ but it is independent from $n$. We can estimate $\left.p_{\perp, n}\right|_{\left\{x_{3}=1\right\}}$ in the same way. Hence, from (4.7) we get

$$
\left|\left\langle\tau_{w, n}\right\rangle_{K_{n}}\right| \leq C \frac{4}{\sqrt[4]{3}}\left(\frac{3}{4}\right)^{n}|\Omega| .
$$

Therefore $\left\langle\tau_{w, n}\right\rangle_{K_{n}} \rightarrow 0$ as $n \rightarrow+\infty$, thus proving that the average wall shear stress decreases if we increase the approximation of the pre-fractal $n$. 
Remark 4.2. In the proof of Theorem 4.9 in [27], it is shown that $\tilde{p}_{n}$ is equibounded in $L^{2}(Q)$. Hence, it seems reasonable to suppose in Theorem 4.1 that $\left\|\tilde{p}_{n}\left(0,0, x_{3}\right)\right\|_{H^{1-\beta}(Q)}$ is equibounded; indeed, in our case $\tilde{p}_{n}\left(0,0, x_{3}\right)$ depends only on the $x_{3}$ variable, which takes values in $I=[0,1]$.

Remark 4.3. It is worth noticing that the hypotheses on the structure of the velocity and pressure fields (which are physically reasonable in laminar pipe flows) are crucial in order to enforce the balance between the pressure field and the wall shear stress. Only in the latter case the stress on $\Omega_{n} \times\{0\}$ and $\Omega_{n} \times\{1\}$ is fixed by the pressure value, and viscous contributions vanish. However, laminar pipe flows are present in several interesting scenery, e.g. blog flow in capillaries and microfluidic devices, making the theoretical results of potential interest for many practical applications.

\section{Numerical approximation}

We now consider the numerical approximation of problem $\left(P_{n}\right)$ by FEM in space and FD in time. We construct an ad hoc mesh which takes into account the singularities of $\mathbf{u}_{n}$ in the vertices and in the edges of $\partial Q_{n}$, in order to obtain an optimal a priori error estimate.

In [6], the case of two-dimensional pre-fractal domains was studied. By adapting the results of [6] to the present case, we first construct a mesh of size $h$ for the twodimensional domains $\Omega_{n}$, and then following [3], we extend it to the 3D case. We point out that any mesh generated by applying the proposed algorithm is compliant with the Grisvard conditions [17].

Let $\{T\}$ be a regular triangulation of $\Omega_{n}, \tilde{\mu} \in[0,1)$ be the grading parameter, $r_{T}$ be the distance of the triangle $T$ from the re-entrant corner of opening $\frac{4 \pi}{3}$, i.e.

$$
r_{T}=\inf _{\left(x_{1}, x_{2}\right) \in T} \sqrt{x_{1}^{2}+x_{2}^{2}}
$$

and $h_{T}$ be the diameter of $T$. We assume that

$$
h_{T} \leq \begin{cases}\tilde{\lambda} h^{\frac{1}{1-\tilde{\mu}}} & \text { for } r_{T}=0, \\ \tilde{\lambda} h r_{T}^{\tilde{\mu}} & \text { for } 0<r_{T} \leq R, \\ \tilde{\lambda} h & \text { for } r_{T}>R,\end{cases}
$$

for some constant $R>0$, where $\tilde{\lambda}$ is the regularity constant of the mesh.

We now extend this two-dimensional mesh in the third dimension by using a uniform mesh size $h$. Let $\{E\}$ be an element of the three-dimensional mesh obtained in such 
way; we denote by $r_{E}$ the distance of $E$ from the edges and by $h_{1, E}$ the length of the projection of $E$ on the $x_{1}$ axis (analogously, we define $h_{2, E}$ and $h_{3, E}$ ). Then the element sizes satisfy the following:

$$
h_{1, E}, h_{2, E} \leq\left\{\begin{array}{ll}
\tilde{\lambda} h^{\frac{1}{1-\tilde{\mu}}} & \text { for } r_{E}=0, \\
\tilde{\lambda} h r_{E}^{\tilde{\mu}} & \text { for } 0<r_{E} \leq R, \\
\tilde{\lambda} h & \text { for } r_{E}>R,
\end{array} \quad h_{3, E} \leq \tilde{\lambda} h .\right.
$$

We denote by $T_{n, h}=\{E\}$ the three-dimensional mesh obtained in this way and by $F$ the faces of the elements. We point out that following this construction we obtain either a pentahedral or a tetrahedral triangulation. In view of the numerical simulations, we choose the pentahedral one, but the results of this section hold also for tetrahedral meshes. The mesh obtained by using this procedure is shown in Figure 2.

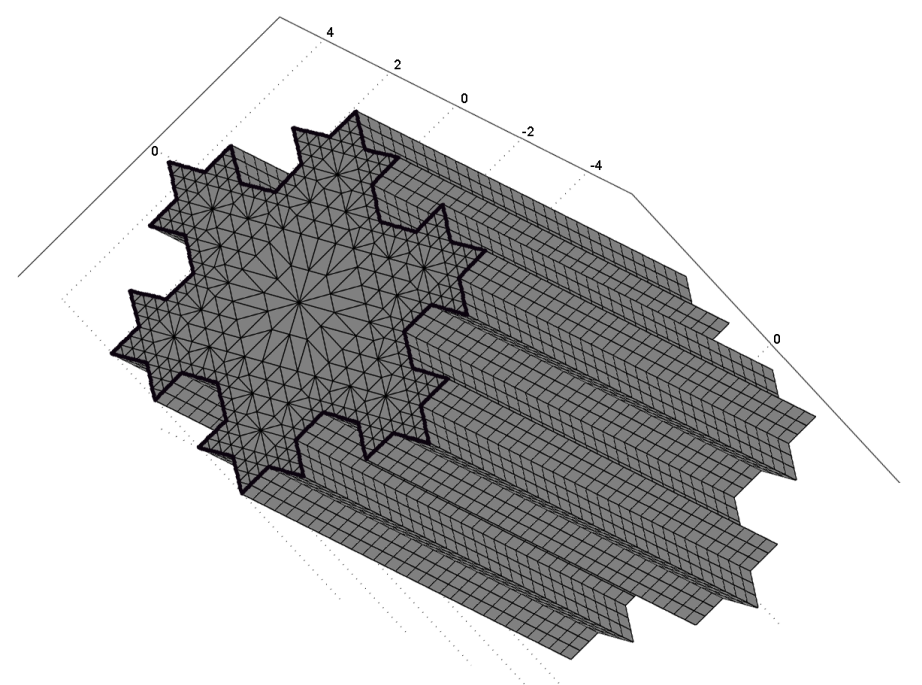

Figure 2: The mesh of $Q_{n}$, for $n=2$.

We now discretize problem $\left(P_{n}\right)$ in space first. Let $\mathbb{P}_{1}$ denote the space of polynomial functions of degree one. We denote by $X_{n, h}$ the finite element space

$$
X_{n, h}=\left\{\mathbf{v} \in L^{2}\left(Q_{n}\right)^{3}:\left.\mathbf{v}\right|_{E} \in\left(\mathbb{P}_{1} \oplus \operatorname{span}\left\{x_{3}^{2}\right\}\right)^{3} \forall E \in T_{n, h} \text { and } \int_{F}[\mathbf{v}]=0 \forall F\right\},
$$

where $[\mathbf{v}]$ denotes the jump of $\mathbf{v}$ on the faces $F$; for boundary faces $[\mathbf{v}]$ is identified with $\mathbf{v}$. 
We denote by $M_{n, h}$ the subspace of piecewise constant functions

$$
M_{n, h}=\left\{q \in L^{2}\left(Q_{n}\right):\left.q\right|_{E} \in \mathbb{P}_{0} \forall E \in T_{n, h} \text { and } \int_{Q_{n}} q=0\right\} .
$$

We point out that $X_{n, h} \nsubseteq H_{0}^{1}\left(Q_{n}\right)^{3}$. Moreover, we equip $X_{n, h}$ with the following norm:

$$
\|\mathbf{u}\|_{X_{n, h}}^{2}:=\sum_{E} \int_{E} \nabla \mathbf{u} \cdot \nabla \mathbf{u} \mathrm{d} \mathcal{L} .
$$

We denote by $I_{n, h}$ the Crouzeix-Raviart interpolant polynomial (see [14]) $I_{n, h}: H_{0}^{1}\left(Q_{n}\right)^{3} \rightarrow X_{n, h}$ defined elementwise by

$$
\int_{F} \mathbf{u} \mathrm{d} \mathcal{S}=\int_{F} I_{n, h} \mathbf{u} \mathrm{d} \mathcal{S} \quad \forall F \subset \partial E, \forall E \in T_{n, h} .
$$

In [2] it is proved that $I_{n, h}$ is well defined. Moreover, it is stable in $H_{0}^{1}\left(Q_{n}\right)^{3}$, i.e.

$$
\int_{E} \nabla\left(I_{n, h} \mathbf{u}\right) \mathrm{d} \mathcal{L} \leq C \int_{E} \nabla \mathbf{u} \mathrm{d} \mathcal{L} \quad \forall E \in T_{n, h} .
$$

If $\mathbf{u}_{n}=\left(u_{1, n}, u_{2, n}, u_{3, n}\right)$ is the solution of $\left(P_{n}\right)$, from (3.3) and Theorem 5.1 in [2] we obtain that

$$
\left\|u_{i, n}-I_{n, h} u_{i, n}\right\|_{X_{n, h}} \leq C h\left\|\mathbf{f}_{n}\right\|_{L^{2}\left(Q_{n}\right)^{3}} .
$$

We now introduce the following mean value operator:

$$
\mathcal{M}_{h, E} p=\frac{1}{\operatorname{meas}(E)} \int_{E} p \mathrm{~d} \mathcal{L} .
$$

We set $\mathcal{M}_{h} p(x):=\mathcal{M}_{h, E} p(x)$ if $x \in E$. By proceeding as in [3], if $p_{n}$ is the pressure associated to the solution $\mathbf{u}_{n}$ of $\left(P_{n}\right)$, from (3.3) we have that

$$
\left\|p_{n}-\mathcal{M}_{h} p_{n}\right\|_{L^{2}\left(Q_{n}\right)} \leq C h\left\|\mathbf{f}_{n}\right\|_{L^{2}\left(Q_{n}\right)^{3}}
$$

The semi-discrete approximation problem reads as follows: given $\mathbf{u}_{n, h}^{0} \in X_{n, h}$, for a.e. $t \in[0, T)$ find $\mathbf{u}_{n, h} \in X_{n, h}$ and $p_{n, h} \in M_{n, h}$ such that

$$
\left(P_{n, h}\right) \begin{cases}\frac{\partial}{\partial t}\left(\mathbf{u}_{n, h}, \mathbf{v}_{h}\right)_{L^{2}\left(Q_{n}\right)}+a_{n}\left(\mathbf{u}_{n, h}, \mathbf{v}_{n, h}\right)-\left(p_{n, h}, \operatorname{div} \mathbf{v}_{h}\right)_{L^{2}\left(Q_{n}\right)}=\left(\mathbf{f}_{n}, \mathbf{v}_{h}\right)_{L^{2}\left(Q_{n}\right)} & \forall \mathbf{v}_{h} \in X_{n, h}, \\ \left(q_{h}, \operatorname{div} \mathbf{u}_{n, h}\right)_{L^{2}\left(Q_{n}\right)}=0 & \forall q_{h} \in M_{n, h}, \\ \mathbf{u}_{n, h}(0)=\mathbf{u}_{n, h}^{0} . & \end{cases}
$$


Problem $\left(P_{n, h}\right)$ is a system of ordinary differential equations. It admits a unique weak solution given by the couple $\left(\mathbf{u}_{n, h}, p_{n, h}\right)$, see e.g. [15].

From Lemma 3.1 in [3], we have that the following inf-sup condition holds: for every $q_{h} \in M_{n, h}$ there exists a function $\mathbf{v}_{h} \in X_{n, h}$, with $\mathbf{v}_{h} \neq 0$, such that

$$
\left(q_{h}, \operatorname{div} \mathbf{v}_{h}\right)_{L^{2}\left(Q_{n}\right)} \geq C\left\|q_{h}\right\|_{L^{2}\left(Q_{n}\right)}\left\|\mathbf{v}_{h}\right\|_{X_{n, h}}
$$

where $C$ is a constant depending on $Q_{n}$ and independent from $h$.

Using the above results, we obtain the following optimal a priori error estimate (see Theorem 3.1 in [3]).

Theorem 5.1. Let $\left(\mathbf{u}_{n}, p_{n}\right)$ be the solution of problem $\left(P_{n}\right)$ and its associated pressure respectively. Let $\left(\mathbf{u}_{n, h}, p_{n, h}\right)$ be the semi-discrete solution of problem $\left(P_{n, h}\right)$. If the grading parameter of the mesh $\tilde{\mu}$ is such that $\tilde{\mu}<\lambda$, where $\lambda$ is given by (3.2), then for a.e. $t \in[0, T)$ the following estimate holds with a constant $C_{1}$ independent from $h$ :

$$
\left\|\mathbf{u}_{n}-\mathbf{u}_{n, h}\right\|_{X_{n, h}}+\left\|p_{n}-p_{n, h}\right\|_{L^{2}\left(Q_{n}\right)} \leq\left\|\mathbf{u}_{n}^{0}-\mathbf{u}_{n, h}^{0}\right\|_{X_{n, h}}+C_{1} h\left\|\mathbf{f}_{n}\right\|_{L^{2}\left(Q_{n}\right)^{3}}
$$

We now approximate problem $\left(P_{n, h}\right)$ in time by using the implicit Euler method. From now on we suppose that $\frac{\partial \mathbf{f}_{n}}{\partial t} \in L^{2}\left(Q_{n}\right)^{3}$.

Let $\Delta t=\frac{T}{N}$ be the time step size, for some integer $N$, and let $t_{l}=l \Delta t$, for $l=$ $0,1, \ldots, N$. We set

$$
V_{n, h}:=\left\{\mathbf{v}_{h} \in X_{n, h}: \int_{Q_{n}} q_{h} \operatorname{div} \mathbf{v}_{h} \mathrm{~d} \mathcal{L}=0 \quad \forall q_{h} \in M_{n, h}\right\} .
$$

Let $\mathcal{P}_{n, h}: L^{2}\left(Q_{n}\right)^{3} \rightarrow V_{n, h}$ be the orthogonal projection. We define $A_{n, h}:=-\mathcal{P}_{n, h} \Delta$ the discrete version of the Stokes operator. Since the restriction of $A_{n, h}$ to $V_{n, h}$ is invertible, and its inverse $A_{n, h}^{-1}$ is positive and self-adjoint, we can define the following discrete norms for $r \in \mathbb{R}$ and $\mathbf{v}_{h} \in V_{n, h}$ :

$$
\left\|\mathbf{v}_{h}\right\|_{r}:=\left\|A_{n, h}^{\frac{r}{2}} \mathbf{v}_{h}\right\|_{L^{2}\left(Q_{n}\right)^{3}} .
$$

We point out that the following hold:

$$
\left\|\mathbf{v}_{h}\right\|_{0}=\left\|\mathbf{v}_{h}\right\|_{L^{2}\left(Q_{n}\right)^{3}}, \quad\left\|\mathbf{v}_{h}\right\|_{1}=\left\|\mathbf{v}_{h}\right\|_{X_{n, h}}, \quad\left\|\mathbf{v}_{h}\right\|_{2}=\left\|A_{n, h} \mathbf{v}_{h}\right\|_{L^{2}\left(Q_{n}\right)^{3}} .
$$


The fully discretized problem reads as follows: setting $\mathbf{u}_{n, h}^{l=0}=\mathbf{u}_{n, h}(0)$, find $\mathbf{u}_{n, h}^{l} \in X_{n, h}$ and $p_{n, h}^{l} \in M_{n, h}$ such that

$$
\left(P_{n, h}^{l}\right) \begin{cases}\frac{\left(\mathbf{u}_{n, h}^{l}-\mathbf{u}_{n, h}^{l-1}, \mathbf{v}_{h}\right)_{L^{2}\left(Q_{n}\right)}}{\Delta t}+a_{n}\left(\mathbf{u}_{n, h}^{l}, \mathbf{v}_{h}\right)-\left(p_{n, h}^{l}, \operatorname{div} \mathbf{v}_{h}\right)_{L^{2}\left(Q_{n}\right)}=\left(\mathbf{f}_{n}\left(t_{l}\right), \mathbf{v}_{h}\right)_{L^{2}\left(Q_{n}\right)} & \forall \mathbf{v}_{h} \in X_{n, h}, \\ \left(q_{h}, \operatorname{div} \mathbf{u}_{n, h}^{l}\right)_{L^{2}\left(Q_{n}\right)}=0 & \forall q_{h} \in M_{n, h} .\end{cases}
$$

We adapt the results of [18] to our case; for the case of Crank-Nicolson, we refer to [19]. From now on, we set

$$
d_{t} \mathbf{w}_{n, h}^{l}=\frac{\mathbf{w}_{n, h}^{l}-\mathbf{w}_{n, h}^{l-1}}{\Delta t} .
$$

Theorem 5.2 (Stability). The fully discretized solution $\mathbf{u}_{n, h}^{l}$ of $\left(P_{n, h}^{l}\right)$ satisfies the following estimate for every $m=0, \ldots, N$ :

$$
\left\|\mathbf{u}_{n, h}^{m}\right\|_{X_{n, h}}^{2}+\Delta t \sum_{l=1}^{m}\left\|\mathbf{u}_{n, h}^{l}\right\|_{2}^{2} \leq\left\|\mathbf{u}_{n, h}^{0}\right\|_{X_{n, h}}^{2}+C \sup _{t \in[0, T]}\left\|\mathbf{f}_{n}(t)\right\|_{L^{2}\left(Q_{n}\right)^{3}}^{2}
$$

where $C$ depends on $T$ and it is independent from $\Delta t, N$ and $h$.

Proof. We take $v_{h}=2 \Delta t A_{n, h} \mathbf{u}_{n, h}^{l}$ as test function in the first equation of $\left(P_{n, h}^{l}\right)$. By using vectorial identities and the properties of $A_{n, h}$, we have

$\left\|\mathbf{u}_{n, h}^{l}\right\|_{1}^{2}-\left\|\mathbf{u}_{n, h}^{l-1}\right\|_{1}^{2}+\Delta t^{2}\left\|d_{t} \mathbf{u}_{n, h}^{l}\right\|_{1}^{2}+2 \Delta t\left\|A_{n, h} \mathbf{u}_{n, h}^{l}\right\|_{L^{2}\left(Q_{n}\right)^{3}}^{2}=2 \Delta t\left(\mathbf{f}_{n}\left(t_{l}\right), A_{n, h} \mathbf{u}_{n, h}^{l}\right)_{L^{2}\left(Q_{n}\right)}$.

From Cauchy-Schwarz and Young inequalities it follows that

$$
\left\|\mathbf{u}_{n, h}^{l}\right\|_{1}^{2}-\left\|\mathbf{u}_{n, h}^{l-1}\right\|_{1}^{2}+\Delta t\left\|A_{n, h} \mathbf{u}_{n, h}^{l}\right\|_{L^{2}\left(Q_{n}\right)^{3}}^{2} \leq \Delta t\left\|\mathbf{f}_{n}\left(t_{l}\right)\right\|_{L^{2}\left(Q_{n}\right)^{3}}^{2} .
$$

By summing on $l$ from 1 to $m$ and taking the supremum for $t \in[0, T]$ on the right-hand side we get the thesis.

We set $\sigma(t)=\min \{1, t\}, \mathbf{e}_{n, h}^{l}:=\mathbf{u}_{n, h}^{l}-\mathbf{u}_{n, h}\left(t_{l}\right)$ and $\eta_{n, h}^{l}:=p_{n, h}^{l}-p_{n, h}\left(t_{l}\right)$ for every $l=1, \ldots, N$. By calculating $\left(P_{n, h}\right)$ in $t=t_{l}$ and by subtracting it to $\left(P_{n, h}^{l}\right)$ we get

$$
\begin{cases}\left(d_{t} \mathbf{e}_{n, h}^{l}, \mathbf{v}_{h}\right)_{L^{2}\left(Q_{n}\right)}+a_{n}\left(\mathbf{e}_{n, h}^{l}, \mathbf{v}_{n, h}\right)-\left(\eta_{n, h}^{l}, \operatorname{div} \mathbf{v}_{h}\right)_{L^{2}\left(Q_{n}\right)}=\left(E_{n, h}^{l}, \mathbf{v}_{h}\right)_{L^{2}\left(Q_{n}\right)} & \forall \mathbf{v}_{h} \in X_{n, h}, \\ \left(q_{h}, \operatorname{div} \mathbf{e}_{n, h}^{l}\right)_{L^{2}\left(Q_{n}\right)}=0 & \forall q_{h} \in M_{n, h},\end{cases}
$$

where the "residual term" $E_{n, h}^{l}$ is defined as follows:

$$
\left(E_{n, h}^{l}, \mathbf{v}_{h}\right)_{L^{2}\left(Q_{n}\right)}:=\frac{1}{\Delta t} \int_{t_{l-1}}^{t_{l}}\left(t-t_{l-1}\right)\left(\frac{\partial^{2} \mathbf{u}_{n, h}(t)}{\partial t^{2}}, \mathbf{v}_{h}\right)_{L^{2}\left(Q_{n}\right)} \mathrm{d} t
$$


We recall an a priori estimate for the semi-discrete solution $\mathbf{u}_{n, h}$ of $\left(P_{n, h}\right)$. For the proof, we refer to Proposition 3.2 in [19].

Proposition 5.3. Let $\mathbf{u}_{n, h}$ be the solution of $\left(P_{n, h}\right)$. Let us suppose that

$$
\left\|\frac{\partial \mathbf{f}_{n}}{\partial t}\right\|_{L^{2}\left(Q_{n}\right)^{3}}^{2} \leq \tilde{C}
$$

where $\tilde{C}$ is independent from $n$ and $h$. Then

$$
\sum_{r=0}^{2} \sigma^{r}(t)\left\|\frac{\partial \mathbf{u}_{n, h}}{\partial t}\right\|_{r}^{2} \leq C\left(\left\|\mathbf{u}_{n, h}(0)\right\|_{X_{n, h}}^{2}+\int_{0}^{t}\left(\left\|\mathbf{f}_{n}\right\|_{L^{2}\left(Q_{n}\right)^{3}}^{2}+\left\|\frac{\partial \mathbf{f}_{n}}{\partial t}\right\|_{L^{2}\left(Q_{n}\right)^{3}}^{2}\right) \mathrm{d} s\right) .
$$

We prove some preliminary lemmas. For the sake of completeness, we give sketches of the proofs.

Lemma 5.4. Let us suppose that

$$
\left\|\frac{\partial \mathbf{f}_{n}}{\partial t}\right\|_{L^{2}\left(Q_{n}\right)^{3}}^{2}+\left\|\frac{\partial^{2} \mathbf{f}_{n}}{\partial t^{2}}\right\|_{L^{2}\left(Q_{n}\right)^{3}}^{2} \leq \tilde{C}
$$

where $\tilde{C}$ is independent from $n$ and $h$. Under the assumptions of Theorem 5.2, the 
residual term $E_{n, h}^{l}$ satisfies the following estimates:

$$
\Delta t \sum_{l=1}^{m}\left\|A_{n, h}^{-1} \mathcal{P}_{n, h} E_{n, h}^{l}\right\|_{L^{2}\left(Q_{n}\right)^{3}}^{2} \leq C \Delta t^{2}\left(\left\|\mathbf{u}_{n, h}(0)\right\|_{X_{n, h}}^{2}+\int_{0}^{T}\left(\left\|\mathbf{f}_{n}\right\|_{L^{2}\left(Q_{n}\right)^{3}}^{2}+\left\|\frac{\partial \mathbf{f}_{n}}{\partial t}\right\|_{L^{2}\left(Q_{n}\right)^{3}}^{2}\right) \mathrm{d} s\right),
$$

$\Delta t \sum_{l=1}^{m}\left\|A_{n, h}^{-\frac{1}{2}} \mathcal{P}_{n, h} E_{n, h}^{l}\right\|_{L^{2}\left(Q_{n}\right)^{3}}^{2} \leq C \Delta t\left(\left\|\mathbf{u}_{n, h}(0)\right\|_{X_{n, h}}^{2}+\int_{0}^{T}\left(\left\|\mathbf{f}_{n}\right\|_{L^{2}\left(Q_{n}\right)^{3}}^{2}+\left\|\frac{\partial \mathbf{f}_{n}}{\partial t}\right\|_{L^{2}\left(Q_{n}\right)^{3}}^{2}\right) \mathrm{d} s\right)$,

$\Delta t \sum_{l=1}^{m} \sigma\left(t_{l}\right)\left\|A_{n, h}^{-\frac{1}{2}} \mathcal{P}_{n, h} E_{n, h}^{l}\right\|_{L^{2}\left(Q_{n}\right)^{3}}^{2} \leq C \Delta t^{2}\left(\left\|\mathbf{u}_{n, h}(0)\right\|_{X_{n, h}}^{2}+\int_{0}^{T}\left(\left\|\mathbf{f}_{n}\right\|_{L^{2}\left(Q_{n}\right)^{3}}^{2}+\left\|\frac{\partial \mathbf{f}_{n}}{\partial t}\right\|_{L^{2}\left(Q_{n}\right)^{3}}^{2}\right) \mathrm{d} s\right)$,

$\sigma^{3}\left(t_{m}\right)\left\|E_{n, h}^{m}\right\|_{L^{2}\left(Q_{n}\right)^{3}}^{2}+\Delta t \sum_{l=2}^{m} \sigma^{2}\left(t_{l}\right)\left\|E_{n, h}^{l}\right\|_{L^{2}\left(Q_{n}\right)^{3}}^{2} \leq C \Delta t^{2}\left\|\mathbf{u}_{n, h}(0)\right\|_{X_{n, h}}^{2}$

$+C \Delta t^{2} \int_{0}^{T}\left(\left\|\mathbf{f}_{n}\right\|_{L^{2}\left(Q_{n}\right)^{3}}^{2}+\left\|\frac{\partial \mathbf{f}_{n}}{\partial t}\right\|_{L^{2}\left(Q_{n}\right)^{3}}^{2}\right) \mathrm{d} s, \quad m=2, \ldots, N$,

$\Delta t \sum_{l=3}^{m} \sigma^{3}\left(t_{l}\right)\left\|A_{n, h}^{-\frac{1}{2}} \mathcal{P}_{n, h} d_{t} E_{n, h}^{l}\right\|_{L^{2}\left(Q_{n}\right)^{3}}^{2} \leq C \Delta t^{2}\left\|\mathbf{u}_{n, h}(0)\right\|_{X_{n, h}}^{2}$

$+C \Delta t^{2} \int_{0}^{T}\left(\left\|\mathbf{f}_{n}\right\|_{L^{2}\left(Q_{n}\right)^{3}}^{2}+\left\|\frac{\partial \mathbf{f}_{n}}{\partial t}\right\|_{L^{2}\left(Q_{n}\right)^{3}}^{2}+\left\|\frac{\partial^{2} \mathbf{f}_{n}}{\partial t^{2}}\right\|_{L^{2}\left(Q_{n}\right)^{3}}^{2}\right) \mathrm{d} s, \quad m=3, \ldots, N$.

For the proof we refer to [18, Lemma 6.1] with small suitable changes.

Lemma 5.5. Under the assumptions of Lemma 5.4, the following inequality holds for every $m=1, \ldots, N$ :

$$
\begin{aligned}
& \left\|\mathbf{e}_{n, h}^{m}\right\|_{L^{2}\left(Q_{n}\right)^{3}}^{2}+\Delta t \sum_{l=1}^{m}\left(\Delta t\left\|d_{t} \mathbf{e}_{n, h}^{l}\right\|_{L^{2}\left(Q_{n}\right)^{3}}^{2}+\left\|\mathbf{e}_{n, h}^{l}\right\|_{1}^{2}\right) \leq C \Delta t\left\|\mathbf{u}_{n, h}(0)\right\|_{X_{n, h}}^{2} \\
& +C \Delta t \int_{0}^{T}\left(\left\|\mathbf{f}_{n}\right\|_{L^{2}\left(Q_{n}\right)^{3}}^{2}+\left\|\frac{\partial \mathbf{f}_{n}}{\partial t}\right\|_{L^{2}\left(Q_{n}\right)^{3}}^{2}\right) \mathrm{d} s .
\end{aligned}
$$


Proof. By taking $\mathbf{v}_{h}=2 \mathbf{e}_{n, h}^{l} \Delta t$ as test function in (5.8), noting that this function belongs to $V_{n, h}$ and using Cauchy-Schwarz and Young inequalities, we get $\left\|\mathbf{e}_{n, h}^{l}\right\|_{L^{2}\left(Q_{n}\right)^{3}}^{2}-\left\|\mathbf{e}_{n, h}^{l-1}\right\|_{L^{2}\left(Q_{n}\right)^{3}}^{2}+\Delta t^{2}\left\|d_{t} \mathbf{e}_{n, h}^{l}\right\|_{L^{2}\left(Q_{n}\right)^{3}}^{2}+c \Delta t\left\|\mathbf{e}_{n, h}^{m}\right\|_{1}^{2} \leq C \Delta t\left\|A_{n, h}^{-\frac{1}{2}} \mathcal{P}_{n, h} E_{n, h}^{l}\right\|_{L^{2}\left(Q_{n}\right)^{3}}^{2}$

By summing from 1 to $N$ and using (5.11), we get the thesis.

Lemma 5.6. Under the assumptions of Lemma 5.4, the following inequality holds for every $m=1, \ldots, N$ :

$$
\begin{aligned}
& \sigma\left(t_{m}\right)\left\|\mathbf{e}_{n, h}^{m}\right\|_{L^{2}\left(Q_{n}\right)^{3}}^{2}+c \Delta t \sum_{l=1}^{m} \sigma\left(t_{l}\right)\left\|\mathbf{e}_{n, h}^{l}\right\|_{1}^{2} \leq C \Delta t^{2}\left\|\mathbf{u}_{n, h}(0)\right\|_{X_{n, h}}^{2} \\
& +C \Delta t^{2} \int_{0}^{T}\left(\left\|\mathbf{f}_{n}\right\|_{L^{2}\left(Q_{n}\right)^{3}}^{2}+\left\|\frac{\partial \mathbf{f}_{n}}{\partial t}\right\|_{L^{2}\left(Q_{n}\right)^{3}}^{2}\right) \mathrm{d} s .
\end{aligned}
$$

Proof. By multiplying (5.16) by $\sigma\left(t_{l}\right)$ and summing on $l$ from 1 to $m$ we get

$$
\sigma\left(t_{m}\right)\left\|\mathbf{e}_{n, h}^{m}\right\|_{L^{2}\left(Q_{n}\right)^{3}}^{2}+c \Delta t \sum_{l=1}^{m} \sigma\left(t_{l}\right)\left\|\mathbf{e}_{n, h}^{l}\right\|_{1}^{2} \leq C \Delta t \sum_{l=1}^{m} \sigma\left(t_{l}\right)\left\|A_{n, h}^{-\frac{1}{2}} \mathcal{P}_{n, h} E_{n, h}^{l}\right\|_{L^{2}\left(Q_{n}\right)^{3}}^{2}
$$

From (5.12) we get the thesis.

Lemma 5.7. Under the assumptions of Lemma 5.4, the following inequality holds for every $m=1, \ldots, N$ :

$$
\begin{aligned}
& \sigma^{2}\left(t_{m}\right)\left\|\mathbf{e}_{n, h}^{m}\right\|_{1}^{2}+c \Delta t \sum_{l=2}^{m} \sigma^{2}\left(t_{l}\right)\left\|A_{n, h} \mathbf{e}_{n, h}^{l}\right\|_{L^{2}\left(Q_{n}\right)^{3}}^{2} \leq C \Delta t^{2}\left\|\mathbf{u}_{n, h}(0)\right\|_{X_{n, h}}^{2} \\
& +C \Delta t^{2} \int_{0}^{T}\left(\left\|\mathbf{f}_{n}\right\|_{L^{2}\left(Q_{n}\right)^{3}}^{2}+\left\|\frac{\partial \mathbf{f}_{n}}{\partial t}\right\|_{L^{2}\left(Q_{n}\right)^{3}}^{2}\right) \mathrm{d} s .
\end{aligned}
$$

Proof. By taking $\mathbf{v}_{h}=2 A_{n, h} \mathbf{e}_{n, h}^{l} \Delta t$ as test function in (5.8), noting that this function belongs to $V_{n, h}$ and using Cauchy-Schwarz and Young inequalities, we get

$$
\left\|\mathbf{e}_{n, h}^{l}\right\|_{1}^{2}-\left\|\mathbf{e}_{n, h}^{l-1}\right\|_{1}^{2}+\Delta t^{2}\left\|d_{t} \mathbf{e}_{n, h}^{l}\right\|_{1}^{2}+c \Delta t\left\|A_{n, h} \mathbf{e}_{n, h}^{l}\right\|_{L^{2}\left(Q_{n}\right)^{3}}^{2} \leq C \Delta t\left\|E_{n, h}^{l}\right\|_{L^{2}\left(Q_{n}\right)^{3}}^{2}
$$

By multiplying the above inequality by $\sigma^{2}\left(t_{l}\right)$, summing on $l$ from 2 to $m$ and using (5.13) we get the thesis. 
By combining (5.17) and (5.18) we obtain the following error estimates for the velocities: for $t_{l} \in(0, T)$

$$
\sigma\left(t_{l}\right)\left\|\mathbf{e}_{n, h}^{l}\right\|_{L^{2}\left(Q_{n}\right)^{3}}^{2}+\sigma^{2}\left(t_{l}\right)\left\|\mathbf{e}_{n, h}^{l}\right\|_{X_{n, h}}^{2} \leq C_{2} \Delta t^{2}\left(\left\|\mathbf{u}_{n, h}(0)\right\|_{X_{n, h}}^{2}+\int_{0}^{T}\left(\left\|\mathbf{f}_{n}\right\|_{L^{2}\left(Q_{n}\right)^{3}}^{2}+\left\|\frac{\partial \mathbf{f}_{n}}{\partial t}\right\|_{L^{2}\left(Q_{n}\right)^{3}}^{2}\right) \mathrm{d} s\right) .
$$

where $C_{2}$ is independent from $t$ and $\Delta t$.

We now focus on obtaining the error estimate for the pressure term $\eta_{n, h}^{l}$.

Theorem 5.8. Under the assumptions of Lemma 5.4, for $l=1, \ldots, N$ we have that $\sigma^{3}\left(t_{l}\right)\left\|\eta_{n, h}^{l}\right\|_{L^{2}\left(Q_{n}\right)}^{2} \leq C \Delta t^{2}\left(\left\|\mathbf{u}_{n, h}(0)\right\|_{X_{n, h}}^{2}+\int_{0}^{T}\left(\left\|\mathbf{f}_{n}\right\|_{L^{2}\left(Q_{n}\right)^{3}}^{2}+\left\|\frac{\partial \mathbf{f}_{n}}{\partial t}\right\|_{L^{2}\left(Q_{n}\right)^{3}}^{2}+\left\|\frac{\partial^{2} \mathbf{f}_{n}}{\partial t^{2}}\right\|_{L^{2}\left(Q_{n}\right)^{3}}^{2}\right) \mathrm{d} s\right)$.

Proof. Taking $\mathbf{v}_{h} \in V_{n, h}$, from (5.8) it follows that

$$
\left(d_{t t} \mathbf{e}_{n, h}^{l}, \mathbf{v}_{h}\right)_{L^{2}\left(Q_{n}\right)}+a_{n}\left(d_{t} \mathbf{e}_{n, h}^{l}, \mathbf{v}_{n, h}\right)=\left(d_{t} E_{n, h}^{l}, \mathbf{v}_{h}\right)_{L^{2}\left(Q_{n}\right)} .
$$

Setting $\mathbf{v}_{h}=2 \Delta t d_{t} \mathbf{e}_{n, h}^{l}$ in the above equation, it follows that

$$
\left\|d_{t} \mathbf{e}_{n, h}^{l}\right\|_{L^{2}\left(Q_{n}\right)^{3}}^{2}-\left\|d_{t} \mathbf{e}_{n, h}^{l-1}\right\|_{L^{2}\left(Q_{n}\right)^{3}}^{2}+c \Delta t\left\|d_{t} \mathbf{e}_{n, h}^{l}\right\|_{1}^{2} \leq C \Delta t\left\|A_{n, h}^{-\frac{1}{2}} \mathcal{P}_{n, h} d_{t} E_{n, h}^{l}\right\|_{L^{2}\left(Q_{n}\right)^{3}}^{2} .
$$

Multiplying the above inequality by $\sigma^{3}\left(t_{l}\right)$, summing on $l$ from 3 to $m$ and using (5.14) we get

$$
\sigma^{3}\left(t_{m}\right)\left\|d_{t} \mathbf{e}_{n, h}^{m}\right\|_{L^{2}\left(Q_{n}\right)^{3}}^{2} \leq C \Delta t^{2}\left(\left\|\mathbf{u}_{n, h}(0)\right\|_{X_{n, h}}^{2}+\int_{0}^{T}\left(\left\|\mathbf{f}_{n}\right\|_{L^{2}\left(Q_{n}\right)^{3}}^{2}+\left\|\frac{\partial \mathbf{f}_{n}}{\partial t}\right\|_{L^{2}\left(Q_{n}\right)^{3}}^{2}+\left\|\frac{\partial^{2} \mathbf{f}_{n}}{\partial t^{2}}\right\|_{L^{2}\left(Q_{n}\right)^{3}}^{2}\right) \mathrm{d} s\right)
$$

We now estimate the residual term $E_{n, h}^{1}$. From direct calculations it follows that

$$
\left\|E_{n, h}^{1}\right\|_{L^{2}\left(Q_{n}\right)^{3}} \leq\left\|\frac{\partial \mathbf{u}_{n, h}\left(t_{1}\right)}{\partial t}\right\|_{L^{2}\left(Q_{n}\right)^{3}}+\frac{1}{\sqrt{\Delta t}}\left(\int_{t_{0}}^{t_{1}}\left\|\frac{\partial \mathbf{u}_{n, h}(t)}{\partial t}\right\|_{L^{2}\left(Q_{n}\right)^{3}}^{2} \mathrm{~d} t\right)^{\frac{1}{2}}
$$

Multiplying the above inequality by $\sigma^{3}\left(t_{1}\right)$, since $\sigma\left(t_{1}\right) \leq \Delta t$, we get

$$
\sigma^{3}\left(t_{1}\right)\left\|E_{n, h}^{1}\right\|_{L^{2}\left(Q_{n}\right)^{3}}^{2} \leq \sigma^{3}\left(t_{1}\right)\left\|\frac{\partial \mathbf{u}_{n, h}\left(t_{1}\right)}{\partial t}\right\|_{L^{2}\left(Q_{n}\right)^{3}}^{2}+\sigma^{2}\left(t_{1}\right) \int_{t_{0}}^{t_{1}}\left\|\frac{\partial \mathbf{u}_{n, h}(t)}{\partial t}\right\|_{L^{2}\left(Q_{n}\right)^{3}}^{2} \mathrm{~d} t .
$$


Since $\sigma^{2}\left(t_{1}\right) \leq \Delta t^{2}$, from Proposition 5.3 we obtain

$$
\sigma^{3}\left(t_{1}\right)\left\|E_{n, h}^{1}\right\|_{L^{2}\left(Q_{n}\right)^{3}}^{2} \leq C \Delta t^{2}\left(\left\|\mathbf{u}_{n, h}(0)\right\|_{X_{n, h}}^{2}+\int_{0}^{T}\left(\left\|\mathbf{f}_{n}\right\|_{L^{2}\left(Q_{n}\right)^{3}}^{2}+\left\|\frac{\partial \mathbf{f}_{n}}{\partial t}\right\|_{L^{2}\left(Q_{n}\right)^{3}}^{2}\right) \mathrm{d} s\right) .
$$

Now, from (5.8), (5.2) and Cauchy Schwarz inequality, we get

$$
\sigma^{3}\left(t_{l}\right)\left\|\eta_{n, h}^{l}\right\|_{L^{2}\left(Q_{n}\right)}^{2} \leq \sigma^{3}\left(t_{l}\right)\left\|d_{t} \mathbf{e}_{n, h}^{l}\right\|_{L^{2}\left(Q_{n}\right)^{3}}^{2}+\sigma^{2}\left(t_{l}\right)\left\|\mathbf{e}_{n, h}^{l}\right\|_{1}^{2}+\sigma^{3}\left(t_{l}\right)\left\|E_{n, h}^{l}\right\|_{L^{2}\left(Q_{n}\right)^{3}}^{2} .
$$

From (5.21), (5.18), (5.13) and (5.22) we get the thesis.

From (5.19), (5.20) and (5.3), the following optimal a priori error estimate follows.

Theorem 5.9. Let $n$ be fixed. Let $\left(\mathbf{u}_{n}(t), p_{n}(t)\right)$ be the solution of problem $\left(P_{n}\right)$ and its associated pressure respectively, and let $\left(\mathbf{u}_{n, h}^{l}, p_{n, h}^{l}\right)$ be the fully discretized solution of $\left(P_{n, h}^{l}\right)$ and its associated pressure respectively. Then for every $l=0,1, \ldots, N$ we have

$$
\begin{aligned}
& \sigma^{2}\left(t_{l}\right)\left\|\mathbf{u}_{n}\left(t_{l}\right)-\mathbf{u}_{n, h}^{l}\right\|_{X_{n, h}}^{2}+\sigma^{3}\left(t_{l}\right)\left\|p_{n}\left(t_{l}\right)-p_{n, h}^{l}\right\|_{L^{2}\left(Q_{n}\right)}^{2} \leq\left\|\mathbf{u}_{n}^{0}-\mathbf{u}_{n, h}^{0}\right\|_{X_{n, h}}^{2} \\
& +C_{1} h^{2}\left\|\mathbf{f}_{n}\right\|_{L^{2}\left(Q_{n}\right)^{3}}^{2}+C_{2} \Delta t^{2} \int_{0}^{T}\left(\left\|\mathbf{f}_{n}\right\|_{L^{2}\left(Q_{n}\right)^{3}}^{2}+\left\|\frac{\partial \mathbf{f}_{n}}{\partial t}\right\|_{L^{2}\left(Q_{n}\right)^{3}}^{2}+\left\|\frac{\partial^{2} \mathbf{f}_{n}}{\partial t^{2}}\right\|_{L^{2}\left(Q_{n}\right)^{3}}^{2}\right) \mathrm{d} s .
\end{aligned}
$$

\section{$6 \quad$ Numerical simulations}

In this section we present two numerical experiments on Stokes flows. We first consider a time-dependent problem, where a quiescent viscous fluid enclosed in a Koch-type prefractal pipe is accelerated by an external field. Secondly, we study stationary Stokes equations, and we aim to numerically validate the theoretical results on the mean shear stress along a fractal boundary, according to Section 4. The simulations have been performed on Comsol V.3.5, on a notebook computer with a Intel quad-Core i5-8250U processor running at $1.60 \mathrm{GHz}$ and equipped with 16 GB RAM.

As to the time dependent problem, we fix $n=3$ and we consider problem $\left(P_{n}\right)$ as follows:

$$
\left(P_{n}\right) \begin{cases}\rho \frac{\partial \mathbf{u}_{n}}{\partial t}(t, x)-\mu \Delta \mathbf{u}_{n}(t, x)+\nabla p_{n}(t, x)=\mathbf{f}_{n}(t, x) & \text { in }[0, T] \times Q_{n}, \\ \operatorname{div} \mathbf{u}_{n}(t, x)=0 & \text { on }[0, T] \times Q_{n}, \\ \mathbf{u}_{n}(t, x)=0 & \text { on }[0, T] \times \partial Q_{n}, \\ \mathbf{u}_{n}(0, x)=\mathbf{u}_{n}^{0}(x) & \text { in } Q_{n},\end{cases}
$$


where in this section $Q_{n}=\Omega_{n} \times[0,2], \rho=1, \mu=0.1, \mathbf{f}_{n}=\left(0,0, \sin \left(\pi x_{1} \sqrt{3}\right)\right), T=1$, $\Delta t=0.01$ and $\mathbf{u}_{n}^{0}(x)=0$.

The behavior of the third component of the velocity field for different time steps in the transient dynamic is described in Figure 3. Since the external force is directed as the $x_{3}$ axis, far from the planes $x_{3}=0$ and $x_{3}=2$ (representing the inlet and the outlet of the fractal pipe) the relevant component of the velocity field is the one aligned in the same direction. On the other hand, in proximity of the bases $\left(\Omega_{n} \times\{0\}\right) \cup\left(\Omega_{n} \times\{2\}\right)$, the velocity component $u_{1, n}$ starts increasing due to the boundary condition $\mathbf{u}_{n}(t, x)=0$ on $\partial Q_{n}$. Thus the resulting motion is represented by an evolving vortex having as axis $x_{2}$.

Due to the dissipative nature of the Stokes operator, the velocity field quickly converges towards a stationary solution, as shown in the last panel of Figure 3. In this case the fluid is quite "trapped" in the green areas of the domain as in Figure 3.

As shown in Section 4, fractal-type boundaries can be useful in Hagen-Poiseuille-like flows, because they enhance the reduction of the mean shear stress exerted by the fluid onto the wall.

More precisely, in Hagen-Poiseuille-like flows, the equations of motion are represented by the stationary Stokes equations, where a pressure difference $\left[p_{n}\right]:=\left.p_{\perp, n}\right|_{\left\{x_{3}=0\right\}}-$ $\left.p_{\perp, n}\right|_{\left\{x_{3}=2\right\}}$ is imposed on the bases $\Omega_{n} \times\{0\}$ and $\Omega_{n} \times\{2\}$. In the stationary case, the pressure drop is balanced by the viscous shear stress exerted on the boundary. Due to the constant cross section, and the pressure condition on the bases, the resulting motion is unidirectional (hence it can be visualized in a generic section of the fractal pipe), and it is mathematically described by a scalar Poisson problem for the Laplace operator with homogeneous Dirichlet boundary conditions for the velocity component $u_{3, n}$. The values of $u_{3, n}$ in a cross section of the pipe are reported in Figure 4 . The different snapshots refer to different values of the parameter $n$.

It turns out that the maximum value of the velocity field is almost constant at each iteration. The specific form of the velocity profile is slightly influenced by the parameter $n$ and, as in the previous numerical example, a "quiescence zone" is detected towards the "trapping blue areas".

Looking at the mean value of the viscous shear stress (Table 1), we notice that $\left\langle\tau_{w, n}\right\rangle$ decreases as $n$ increases, according to Theorem 4.1.

Figure 5 shows the average wall shear stress data collected in Table 1. One may observe the exponential decay given by Theorem 4.1 (more precisely, in accordance with the curve $\left.y=0.22\left(\frac{3}{4}\right)^{n}\right)$. The reader may notice that the first two numerical values of Table 1 does not perfectly match with the exponential curve; this is due to numerical integration errors which are larger for coarse meshes, i.e. $n=1,2$. 
Slice: $\mathbf{x} \_3$-velocity $[\mathrm{m} / \mathrm{s}]$

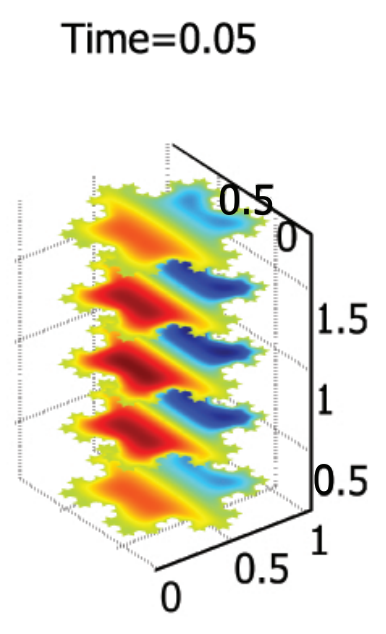

Max: 0.0332

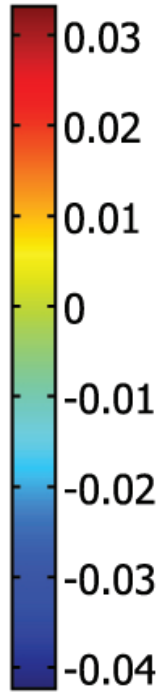

Min: -0.0424

Slice: x_3-velocity [m/s]

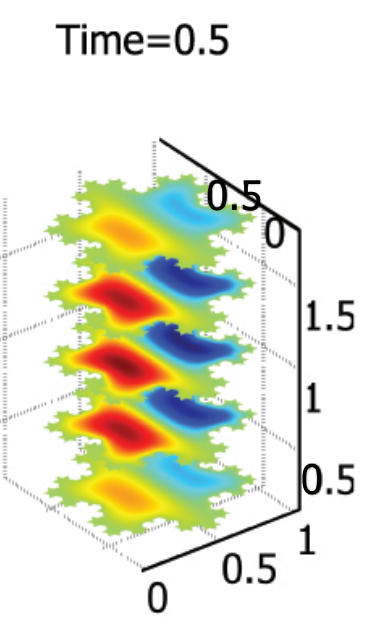

Max: 0.0999

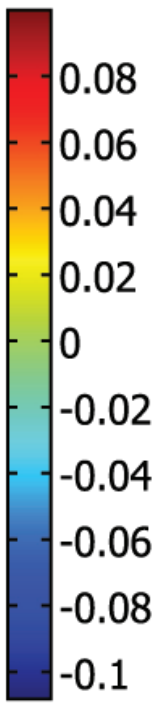

Min: -0.108
Slice: $\mathrm{x} \_3$-velocity [m/s] Max: 0.0723
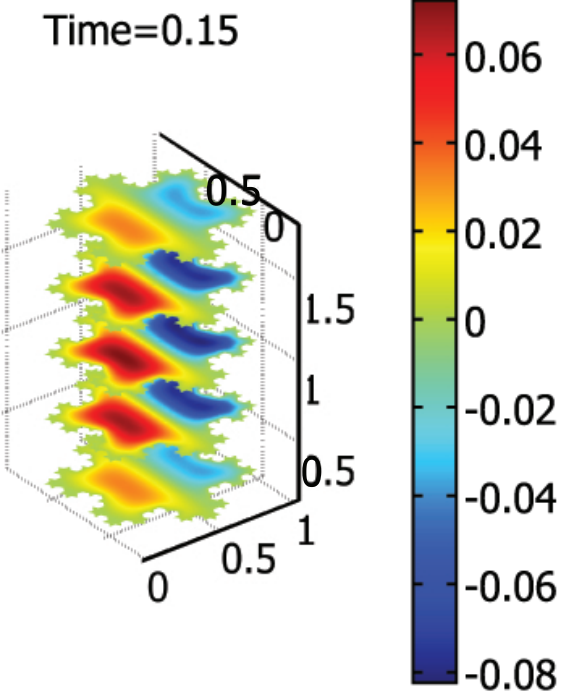

Min: -0.0824

Slice: $\mathrm{x} \_3$-velocity $[\mathrm{m} / \mathrm{s}]$

Max: 0.100

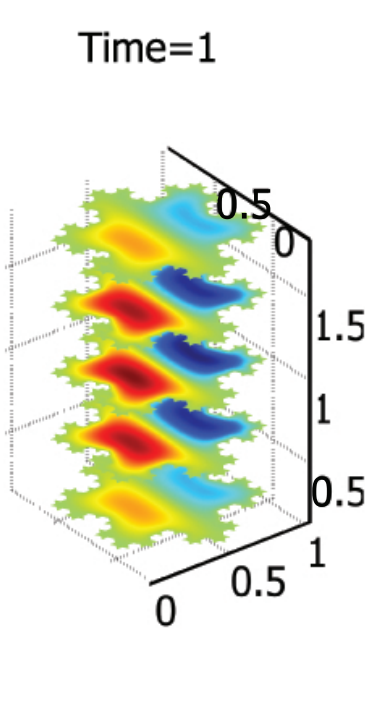

Min: -0.109

Figure 3: Time evolution of the velocity component $u_{3, n}$. The time sequence from the top (left panel) is referring to the time steps $t=0.05, t=0.15, t=0.50$ and $t=1.00$.

Acknowledgements. S. C., M. R. L. and P. V. have been supported by the Gruppo Nazionale per l'Analisi Matematica, la Probabilità e le loro Applicazioni (GNAMPA) of the Istituto Nazionale di Alta Matematica (INdAM). 

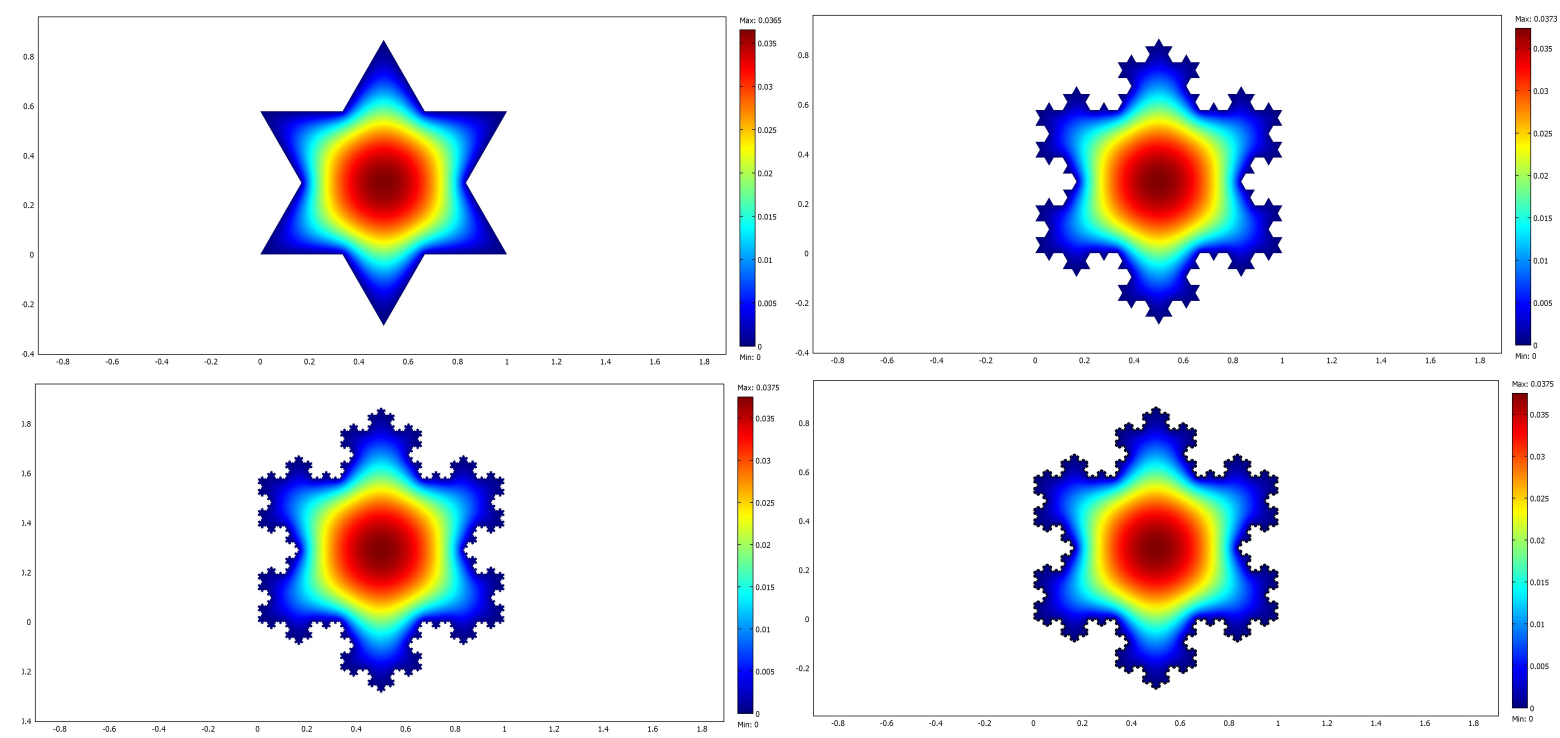

Figure 4: Snapshots of the velocity component $u_{3, n}$ for $n=1,3,5,7$.

\begin{tabular}{c|c}
$n$ & $\left\langle\tau_{w, n}\right\rangle$ \\
\hline 1 & 0,143352 \\
2 & 0,118425 \\
3 & 0,09157 \\
4 & 0,069939 \\
5 & 0,05179 \\
6 & 0,038986 \\
7 & 0,028286
\end{tabular}

Table 1: The values of the mean wall shear stress obtained in our simulations.

\section{References}

[1] E. Akkermans, Statistical mechanics and quantum fields on fractals, Fractal geometry and dynamical systems in pure and applied mathematics. II, Fractals in applied mathematics, Contemp. Math., 601, Amer. Math. Soc., Providence, RI, 2013, 1-21.

[2] T. Apel, S. Nicaise, J. Schöberl, Crouzeix-Raviart type finite elements on anisotropic meshes, Numer. Math., 89 (2001), 2, 193-223.

[3] T. Apel, S. Nicaise, J. Schöberl, A non-conforming finite element method with anisotropic mesh grading for the Stokes problem in domains with edges, IMA J. Numer. Anal., 21 (2001), 4, 843856.

[4] M. Cefalo, S. Creo, M. R. Lancia, P. Vernole, Nonlocal Venttsel' diffusion in fractal-type domains: regularity results and numerical approximation, Math. Methods Appl. Sci., 42 (2019), 14, 47124733. 


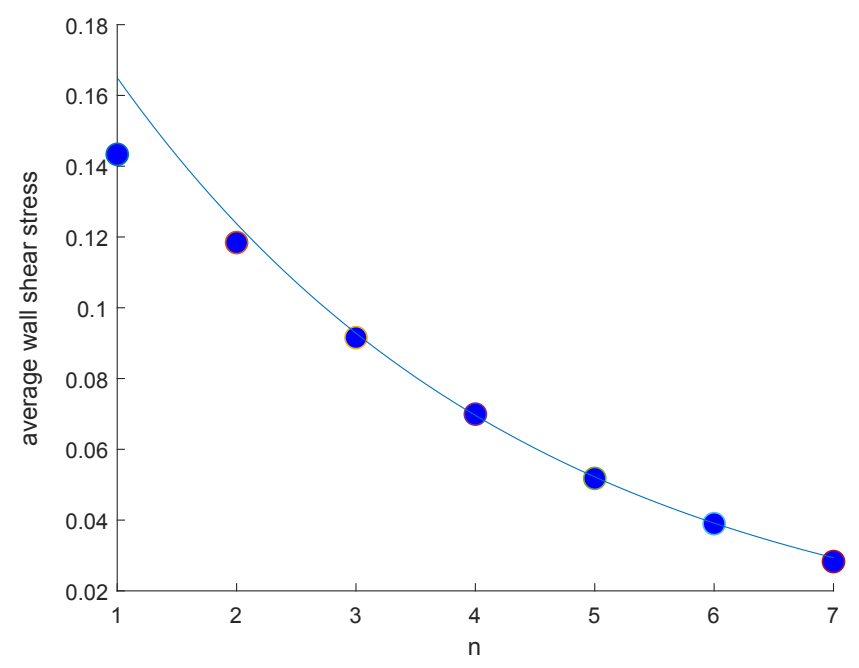

Figure 5: The exponential decay of $\left\langle\tau_{w, n}\right\rangle$.

[5] M. Cefalo, G. Dell'Acqua, M. R. Lancia, Numerical approximation of transmission problems across Koch-type highly conductive layers, Appl. Math. Comput., 218 (2012), 9, 5453-5473.

[6] M. Cefalo, M. R. Lancia, An optimal mesh generation algorithm for domains with Koch type boundaries, Math. Comput. Simulation, 106 (2014), 133-162.

[7] M. Cefalo, M. R. Lancia, H. Liang, Heat-flow problems across fractals mixtures: regularity results of the solutions and numerical approximation, Differential Integral Equations, 26 (2013), 9-10, $1027-1054$.

[8] S. Creo, M. Hinz, M. R. Lancia, A. Teplyaev, P. Vernole, Magnetostatic problems in fractal domains, Fractals and Dynamics in Mathematics, Sciences and the Arts Volume 5: Analysis, Probability and Mathematical Physics on Fractals, World Scientific, 2020, 477-502. DOI: 10.1142/9789811215537_0015. Preprint: https://arxiv.org/abs/1805.08262

[9] S. Creo, M. R. Lancia, Friedrichs inequality in irregular domains, J. Math. Anal. Appl., 484 (2020), 1, 123665.

[10] S. Creo, M. R. Lancia, A. I. Nazarov, P. Vernole, On two-dimensional nonlocal Venttsel' problems in piecewise smooth domains, Discrete Contin. Dyn. Syst. Series S, 12 (2019), 1, 57-64.

[11] S. Creo, M. R. Lancia, A. Vélez-Santiago, P. Vernole, Approximation of a nonlinear fractal energy functional on varying Hilbert spaces, Commun. Pure Appl. Anal., 17 (2018), 2, 647-669.

[12] S. Creo, M. R. Lancia, P. Vernole, Convergence of fractional diffusion processes in extension domains, J. Evol. Equ., 20 (2020), 1, 109-139.

[13] S. Creo, V. Regis Durante, Convergence and density results for parabolic quasi-linear Venttsel' problems in fractal domains, Discrete Contin. Dyn. Syst. Series S, 12 (2019), 1, 65-90.

[14] M. Crouzeix, P. A. Raviart, Conforming and nonconforming finite element methods for solving the stationary Stokes equations, RAIRO Anal. Numér, 7 (1973), 33-75. 
[15] R. Dautray, J. L. Lions, Mathematical Analysis and Numerical Methods for Science and Technology. Vol 6. Evolution Problems II, Springer-Verlag, Berlin, 1993.

[16] K. Falconer, The Geometry of Fractal Sets, Cambridge University Press, Cambridge, 1986.

[17] P. Grisvard, Elliptic Problems in Nonsmooth Domains, Pitman, Boston, 1985.

[18] Y. He, The Euler implicit/explicit scheme for the $2 D$ time-dependent Navier-Stokes equations with smooth or non-smooth initial data, Math. Comp., 77 (2008), 264, 2097-2124.

[19] J. G. Heywood, R. Rannacher, Finite-element approximation of the nonstationary Navier-Stokes problem, Part IV: Error analysis for second-order time discretization, SIAM J. Numer. Anal., $\mathbf{2 7}$ (1990), 2, 353-384.

[20] M. Hinz, L. Rogers, Magnetic fields on resistance spaces, J. Fractal Geometry, 3 (2016), 1, 75-93.

[21] M. Hinz, A. Teplyaev, Dirac and magnetic Schrödinger operators on fractals, J. Funct. Anal., 265 (2013), 11, 2830-2854.

[22] D. Jerison, C. E. Kenig, The inhomogeneous Dirichlet problem in Lipschitz domains, J. Funct. Anal., 130 (1995), 1, 161-219.

[23] A. Jonsson, H. Wallin, Function Spaces on Subsets of $\mathbb{R}^{n}$, Part 1, Math. Reports, vol.2, Harwood Acad. Publ., London, 1984.

[24] T. Kato, Perturbation Theory for Linear Operators, II edit., Springer-Verlag, Berlin-New York, 1976.

[25] M. R. Lancia, A. Vélez-Santiago, P. Vernole, Quasi-linear Venttsel' problems with nonlocal boundary conditions on fractal domains, Nonlinear Anal. Real World Appl., 35 (2017), 265-291.

[26] M. R. Lancia, P. Vernole, Venttsel' problems in fractal domains, J. Evol. Equ., 14 (2014), 3, $681-712$.

[27] M. R. Lancia, P. Vernole, The Stokes problems in fractal domains: Asymptotic behaviour of the solutions, Discrete Contin. Dyn. Syst. Ser. S, 13 (2020), 5, 1553-1565.

[28] S. Monniaux, Navier-Stokes equations in arbitrary domains: The Fujita-Kato scheme, Math. Res. Lett., 13 (2006), 455-461.

[29] S. Nicaise, Regularity of the solutions of elliptic systems in polyhedral domains, Bull. Belg. Math. Soc. Simon Stevin, 4 (1997), 3, 411-429.

[30] A. Quarteroni, A. Valli, Numerical Approximation of Partial Differential Equations, SpringerVerlag, Berlin, 1994.

[31] S. Shen, J. Xu, J. Zhou, Y. Chen, Flow and heat transfer in microchannels with rough wall surface, Energy Convers. Manage., 47 (2006), 1311-1325.

[32] H. Sohr, The Navier-Stokes Equations. An Elementary Functional Analytic Approach, Birkhäuser Verlag, Basel, 2001.

[33] G. G. Stokes, On the theories of the internal friction of fluids in motion, and of the equilibrium and motion of elastic solids, Transactions of the Cambridge Philosophical Society, 8 (1845), $287-341$. 
[34] S. P. Sutera, R. Skalak, The history of Poiseuille's law, Annual review of fluid mechanics, 25, 1-19, Annual Reviews, Palo Alto, CA, 1993.

[35] B. Taylor, A. L. Carrano, S. G. Kandlikar, Characterization of the effect of surface roughness and texture on fluid flow past, present, and future, Int. J. Thermal Sci., 45 (2006), 962-968.

[36] R. Temam, Roger Navier-Stokes Equations. Theory and Numerical Analysis, Studies in Mathematics and its Applications, 2, North-Holland Publishing Co., Amsterdam-New York, 1979.

[37] S. S. Yang, B. Yu, M. Zou, M. Liang, A fractal analysis of laminar flow resistance in roughened microchannels, Int. J. Heat Mass Transf., 77 (2014), 208-217. 